\title{
Design and Construction of an Instrumentation System to Capture the Response of Advanced Materials Impacted by Intense Proton Pulses
}

\author{
F. Carra $(\mathbb{D}$, C. Charrondiere $(\mathbb{D}$, M. Guinchard $(\mathbb{D}$, and O. Sacristan de Frutos \\ CERN, Espl. des Particules 1, 1211 Meyrin, Switzerland \\ Correspondence should be addressed to F. Carra; federico.carra@cern.ch
}

Received 27 July 2020; Revised 10 February 2021; Accepted 16 March 2021; Published 5 April 2021

Academic Editor: Paola Antonaci

Copyright ( 2021 F. Carra et al. This is an open access article distributed under the Creative Commons Attribution License, which permits unrestricted use, distribution, and reproduction in any medium, provided the original work is properly cited.

In recent years, significant efforts were taken at CERN and other high-energy physics laboratories to study and predict the consequences of particle beam impacts on devices such as collimators, targets, and dumps. The quasi-instantaneous beam impact raises complex dynamic phenomena which may be simulated resorting to implicit codes, for what concerns the elastic or elastoplastic solid regime. However, when the velocity of the produced stress waves surpasses the speed of sound and we enter into the shock regime, highly nonlinear numerical tools, called Hydrocodes, are usually necessary. Such codes, adopting very extensive equations of state, are also able to well reproduce events such as changes of phase, spallation, and explosion of the target. In order to derive or validate constitutive numerical models, experiments were performed in the past years at CERN HiRadMat facility. This work describes the acquisition system appositely developed for such experiments, whose main goal is to verify, mostly in real time, the response of matter when impacted by highly energetic proton beams. Specific focus is given to one of the most comprehensive testing campaigns, named "HRMT-14." In this experiment, energy densities with peaks up to $20 \mathrm{~kJ} / \mathrm{cm}^{3}$ were achieved on targets of different materials (metallic alloys, graphite, and diamond composites), by means of power pulses with a population up to $3 \times 1013 \mathrm{p}$ at $450 \mathrm{GeV}$. The acquisition relied on embarked instrumentation (strain gauges, temperature probes, and vacuum sensors) and on remote acquisition devices (laser Doppler vibrometer and high-speed camera). Several studies have been performed to verify the dynamic behaviour of the standard strain gauges and the related cabling in the chosen range of acquisition frequency (few $\mathrm{MHz}$ ). The strain gauge measurements were complemented by velocity measurements performed using a customised long-range laser Doppler vibrometer (LDV) operating in the amplitude range of $24 \mathrm{~m} / \mathrm{s}$; the LDV, together with the high-speed video camera (HSVC), has been placed at a distance of $40 \mathrm{~m}$ from the target to minimize radiation damage. In addition, due to the large number of measuring points, a radiation-hard multiplexer switch has been used during the experiment: this system was designed to fulfil the multiple requirements in terms of bandwidth, contact resistances, high channel reduction, and radiation resistance. Shockwave measurements and intense proton pulse effects on the instrumentation are described, and a brief overlook of the comparison of the results of the acquisition devices with simulations, performed with the finite element tool Autodyn, is given. Generally, the main goal of such experiments is to benchmark and improve material models adopted on the tested materials in explicit simulations of particle beam impact, a design scenario in particle accelerators, performed by means of Autodyn. Simulations based on simplified strain-dependent models, such as Johnson-Cook, are run prior to the experiment. The model parameters are then updated in order to fit the experimental response, under a number of load cases to ensure repeatability of the model. This paper, on the other hand, mostly focuses on the development of the DAQ for HiRadMat experiments, and in particular for HRMT-14. Such development, together with the test design and run, as well as postmortem examination, spanned over two years, and its fundamental results, mostly in terms of dedicated instrumentation, have been used in all successive HiRadMat experiments as of 2014. This experimental method can also find applications for materials undergoing similarly high strain rates and temperature changes (up to $106 \mathrm{~s}-1$ and $10.000 \mathrm{~K}$, respectively), for example, in the case of experiments involving fast and intense loadings on materials and structures. 


\section{Introduction}

The introduction in past years of extremely energetic particle accelerators such as the Large Hadron Collider (LHC) [1] brought about the need for advanced cleaning and protection systems in order to safely increase the energy and intensity of particle beams to unprecedented levels [2]. A key element of the cleaning and protection system is constituted by collimators [3], which are designed to intercept and absorb beam particles and to shield other components from the catastrophic consequences of beam orbit errors [4]. Furthermore, recent ambitious programs for the development of accelerator facilities aimed at the massive production of elusive particles, such as neutrinos or muons, relying on target systems submitted to the impact of proton beams at unprecedented intensities (impact power up to $5 \mathrm{MW}$ ) [5]. Therefore, it is paramount to assess the response to such potentially destructive events of materials presently used, or being developed for future use, in collimators and other beam intercepting devices (targets, dumps, absorbers, spoilers, windows, etc.).

\subsection{Physical Phenomenon and Numerical Simulations.} Complex numerical methods have been developed in the last years to study dynamic phenomena, such as phase transitions, density changes, shockwave propagation, explosions, and fragment projections, generated in matter when it is impacted by highly energetic particle beams. These effects, triggered by the thermomechanical load acting on the target, completely hinder second-order effects such as radiation damage which, for single particle beam pulses, are associated to a negligible amount of displacements per atom (dpa) [6]. Unfortunately, material models required to perform such simulations, at the extreme conditions as to temperature, pressure, and density induced by beam impacts, are hardly available in the scientific literature; besides, most of the existing information is often classified as it is drawn from military research. Finally, very few data can be found for nonconventional alloys and compounds. Figure 1 shows an example of numerical simulation of particle beam impact on an LHC collimator, performed with the explicit tool Autodyn. The simulation is compared with an experimental result observed at the CERN HiRadMat facility [8].

It is well known that the rapid interaction of highly energetic particle beams with matter induces dynamic responses in the impacted structure $[9,10]$. The nature, intensity, and time scale of these responses depend on several parameters, mainly deposited energy and energy density, interaction duration, and physical and mechanical properties of the impacted material.

In order to derive or validate the numerical models necessary to simulate the phenomenon (equations of state, strength models, and failure models), a comprehensive, firstof-its-kind experiment was run at CERN HiRadMat facility in 2012: performed tests entailed the controlled impact of intense and energetic proton pulses on a number of specimens made of six different materials. The preparation of the experiment and its operation took a year.
1.2. CERN HiRadMat Facility. First tests of robustness and damage effects on LHC collimators were performed in ad hoc installations in the TT40 transfer beam line between LHC and CNGS in 2004 and 2006. The difficulty in performing such tests on temporary installations and the potential impact on operating transfer lines was the main motivations for the decision to build a new dedicated facility, called High Radiation to Materials (HiRadMat), designed to study beam impacts on materials and accelerator components [11].

The main beam parameters of HiRadMat facility are listed in Table 1 . The beam spot size at the focal point of the experiment can be varied from 0.5 to $2 \mathrm{~mm}^{2}$; this, together with the variable beam intensity, offers sufficient flexibility to test materials at different deposited energy densities.

Experiments in HiRadMat must comply with strict safety requirements according to the CERN rules and the ALARA principles. In particular, ALARA stands for "As Low As Reasonably Possible" exposition to radioactive environment. To respect this basic principle and to minimize the dose taken during installation and removal of the experiments, the experimental setup had to be confined into a vacuum tank interfaced with the surface through a connection table, Figure 2. The interface permits the remote control of the embarked equipment from the surface.

Services such as water cooling, electrical power, and signal cables are delivered via plug-in connectors so that the experiments can be installed with minimal intervention in the area. A simple or double confinement of the test material must be provided to avoid any spray of melted (sublimated or evaporated) material in the test area and to provide an inert-gas or vacuum environment for the test material.

The experimental setup is first prepared, aligned, and tested on a support plate in a surface laboratory where a test stand identical to those in the experimental area is installed. The whole setup is then lowered via the access shaft in the underground areas, Figure 2.

The setup is then moved with an overhead crane and installed using the plug-in system in one of the three test positions of the experimental area (position B in Figure 3). Limited access to this area may be authorized, depending on the radiation levels, to install auxiliary equipment, such as gas lines, when it is not technically possible via plug-in connectors.

Once the experiment is concluded, the test stand is moved remotely with the overhead crane and positioned in a cool-down area (position C in Figure 3). The cool-down time depends on the irradiated material and on the absorbed dose. Radiation monitors, remotely controlled, are placed in each area, allowing careful planning of all operations with minimal direct intervention.

1.3. Experiment Layout. The instrumentation system described in this paper was designed and built for the HRMT14 experiment, performed in 2012. Thanks to its very good performance, it was then used as the cornerstone also for other successive experiments, such as HRMT-23 "Jaws" [12] and HRMT-36 "Multimat" [13]. The HRMT-14 


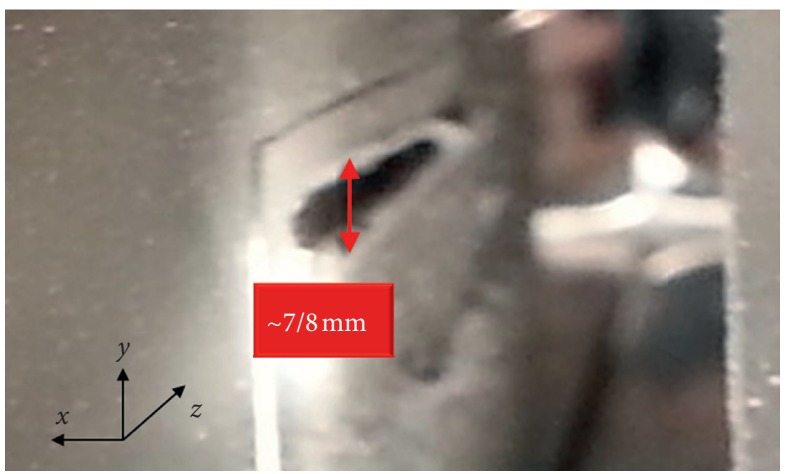

(a)

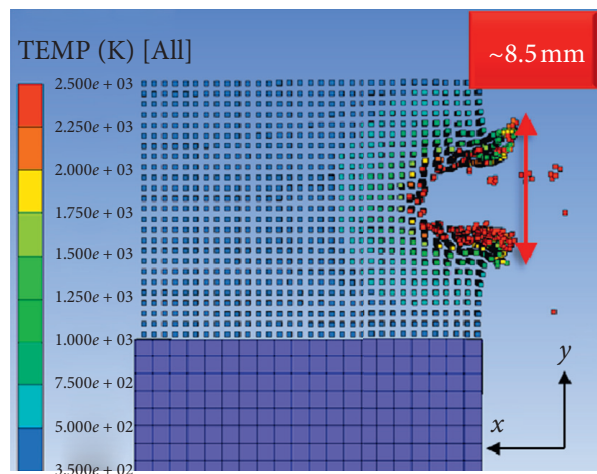

(b)

FiguRe 1: (a) LHC tertiary collimator impacted by 24 proton bunches, with an intensity of $1.3 \times 10^{11} \mathrm{p}$ and energy of $440 \mathrm{GeV}$ [7]. (b) Comparison with a simulation performed with Autodyn and smoothed-particle hydrodynamics (SPH) technique [8]. Beam direction is $Z$.

TABLE 1: HiRadMat beam parameters.

\begin{tabular}{lcc}
\hline & Protons & Ions $\left(\mathrm{Pb}^{82+}\right)$ \\
\hline Energy & $440 \mathrm{GeV}$ & $173.5 \mathrm{GeV} / \mathrm{u}$ \\
Bunch intensity (max) & $1.7 \mathrm{e} 11^{*}$ & $7 \mathrm{e} 9$ \\
Number of bunches (max) & 288 & 52 \\
Pulse intensity (max) & $4.9 \mathrm{e} 13$ & $3.6 \mathrm{e} 9$ \\
Pulse energy (max) & $3.4 \mathrm{MJ}$ & $21 \mathrm{~kJ}$ \\
Bunch length & $11.24 \mathrm{~cm}$ & $11.24 \mathrm{~cm}$ \\
Bunch spacing & $25 / 50 / 75 / 150 \mathrm{~ns}$ & $100 \mathrm{~ns}$ \\
Pulse length & $7.2 \mu \mathrm{s}$ & $5.2 \mu \mathrm{s}$ \\
\hline
\end{tabular}

* Note that, until 2022, the effective bunch intensity will be limited to $1.2 \mathrm{e} 11$ $\mathrm{p}$, and an increase up to the design intensity of $1.7 \mathrm{e} 11 \mathrm{p}$ (and even up to $2.3 \mathrm{e} 11 \mathrm{p}$, which will be feasible after the LIU upgrade implementation) will only be possible as of 2022, and not for a pulse at the maximum number of bunches. In fact, for a pulse with 288 bunches at the intensity $2.3 \mathrm{e} 11 \mathrm{p}$, an upgrade of the HiRadMat beam window and dump would be necessary.

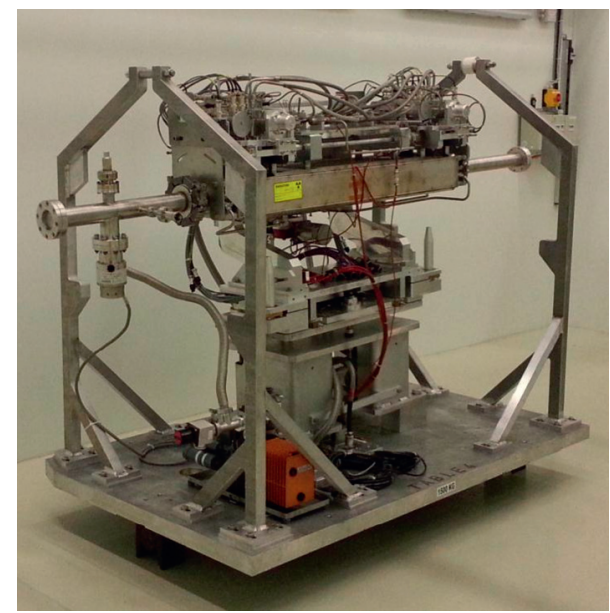

FIgURE 2: 3D view of a test stand: the fixed base (in black), the support table (blue), and the experimental test stand; in this example, an LHC collimator is tested.

experimental setup consisted of a multimaterial sample holder allowing to test six different materials under hadron beams of different intensity (from $1 \times 10^{11}$ to $3 \times 10^{13}$ protons) at the energy of $440 \mathrm{GeV}$. The test-bench was designed and equipped in order to measure in real-time physical quantities necessary to reconstruct the material models, such as axial and hoop strains, radial velocity, and temperature. Data were collected at a sampling rate of $4 \mathrm{MHz}$, to be able to capture the dynamic phenomena without aliasing. In fact, we targeted capturing simulated frequencies up to $500 \mathrm{kHz}$, see Figure 4. Projection of particles generated by the beam impact was filmed by a high-speed camera, with a sampling rate of $20 \mathrm{kfps}$.

1.3.1. Tested Materials. Three of the tested materials were relatively conventional pure metals or metallic alloys: Inermet $^{\circledR} 180$ (tungsten heavy alloy), Glidcop ${ }^{\circledR}$ AL-15 LOX (dispersion-strengthened copper), and Molybdenum. Three novel composites, developed at CERN in past years, were also tested, namely, Molybdenum-Copper-Diamond (MoCuCD), Copper-Diamond (CuCD), and MolybdenumGraphite (MoGR) [14]. Material properties are reported in Table 2. One can notice that the novel materials have in common a lower density with respect to the other three materials. MoGR and CuCD also feature a thermal conductivity close to or above that of pure copper, which favors their cooling by conduction in operation. Their coefficient of thermal expansion is close to that of refractory metals, such as tungsten and molybdenum. Finally, their specific heat is higher than that of the other three materials. All these properties combined together strongly improve their thermomechanical behaviour, increasing the resistance of the novel composites to the particle beam impact.

1.3.2. Test Bench. The test bench was primarily constituted by a vacuum vessel and a specimen housing featuring 12 material samples tiers arranged in two rows of six, Figure 5.

The specimen housing could be accurately positioned via a two-degree-of-freedom actuation system. The $300 \mathrm{~mm}$ vertical travel permitted to align each of the six tiers with the beam axis, while the $120 \mathrm{~mm}$ lateral movement permitted to switch between the two arrays. Two different specimen shapes were chosen for each tested material: cylindrical disks (type 1) for medium intensity tests, to measure axially symmetric shock waves; cylinders with a half-moon cross 


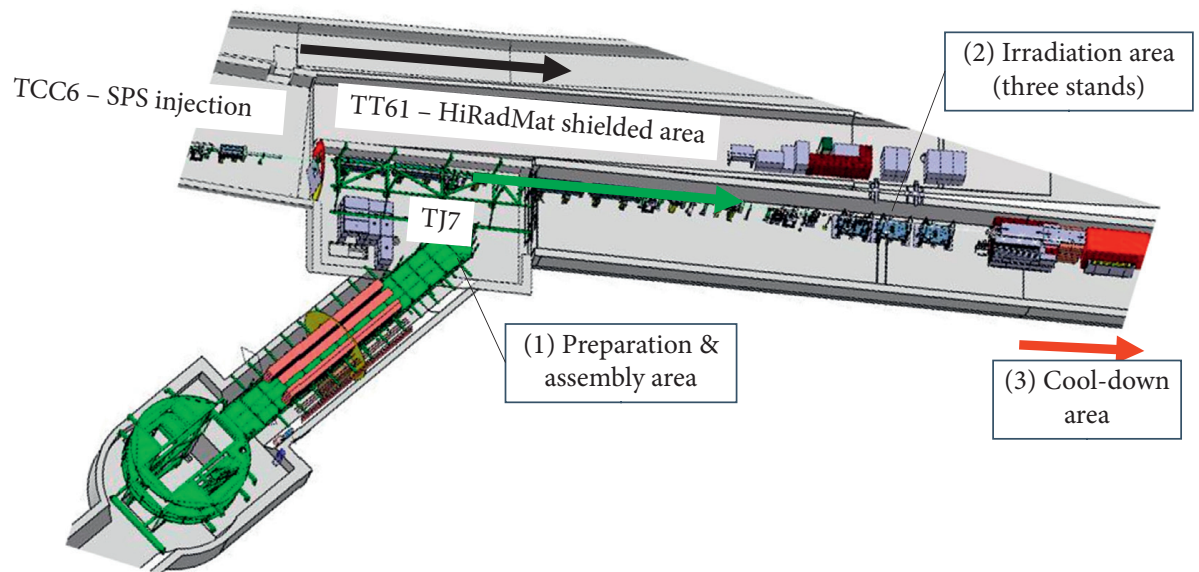

Figure 3: The different stages during the lifetime of an experiment in HiRadMat [11].

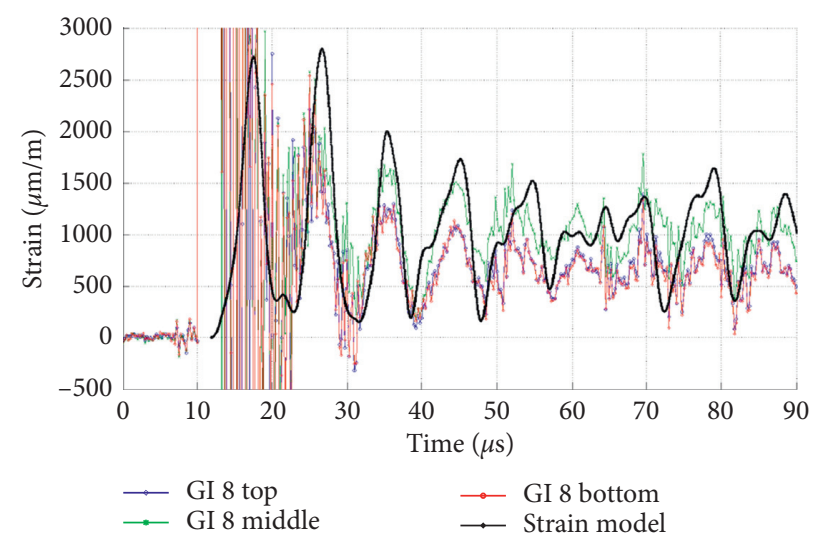

FIgURE 4: Results of the medium-intensity test on Glidcop ${ }^{\circledR}$, hoop strain without smoothing function (blue, green, and red dotted lines), compared with Autodyn simulation (black line).

TABle 2: Properties of materials tested during the experiment.

\begin{tabular}{|c|c|c|c|c|c|c|}
\hline Material & Inermet $^{\circledR} 180$ & Mo & Glidcop ${ }^{\circledR}$ & $\mathrm{MoCuCD}$ & $\mathrm{CuCD}$ & MoGR (a) \\
\hline Density $\left(\mathrm{kg} / \mathrm{m}^{3}\right)$ & 18000 & 10220 & 9800 & 6900 & 5400 & $3700 \div 5300$ \\
\hline Atomic number & 70.8 & 42 & 29 & 17.4 & 11.4 & $13.7 \div 15.9$ \\
\hline Melting temperature $(\mathrm{b})\left({ }^{\circ} \mathrm{C}\right)$ & 1343 & 2623 & 1083 & 1083 & 1083 & 2505 \\
\hline Thermal conductivity $(\mathrm{W} / \mathrm{m} / \mathrm{K})$ & 90 & 138 & 365 & 155 & 490 & $135 \div 320$ \\
\hline CTE $(10-6 \mathrm{~K}-1)$ & 5.25 & 5 & 16.60 & 7 & 7 & $6.80 \div 7.59$ \\
\hline Specific heat $(\mathrm{J} / \mathrm{kg} / \mathrm{K})$ & 150 & 251 & 391 & 360 & 420 & $369 \div 574$ \\
\hline Young's modulus (GPa) & 360 & 330 & 130 & 260 & 220 & $79 \div 179$ \\
\hline Tensile strength (MPa) & 660 & 640 & 413 & 140 & 70 & $53 \div 135$ (c) \\
\hline Poisson's ratio & 0.286 & 0.356 & 0.326 & 0.300 & 0.300 & 0.190 \\
\hline Electrical conductivity (MS/m) & 8.6 & 19.2 & 53.8 & 10 & 12.6 & 1 \\
\hline Sound speed $(\mathrm{m} / \mathrm{s})$ & 4470 & 5700 & 3800 & 6100 & 6400 & $4700 \div 5800$ \\
\hline
\end{tabular}

(a) Three different material grades. (b) Melting temperature of the low-melting phase. (c) Flexural strength, measured with four-point bending method.

(d) On a target in uniaxial stress conditions.

section (type 2) for high intensity tests, allowing extreme surface phenomena (melting, material explosion, debris projections, etc.) to be visualized and optically acquired. The main parameter changing between medium and high intensity tests was the number of bunches per pulse leading, in the case of high intensity tests, to a higher amount of energy absorbed by the target. See, for example, Tables 3 and 4 . The diameter of both sample types is $40 \mathrm{~mm}$, and their length is
$30 \mathrm{~mm}$. The flat surface of type 2 was $2 \mathrm{~mm}$ from the center of the cylinder, Figure 6.

Material specimens were kept in place by graphite restraints, to minimize the propagation of shockwaves into the housing. The number of specimens per tier varied as a function of the radiation and nuclear interaction lengths of the sample material, in order to observe the energy deposition peak in each material from the lightest to the heaviest. 


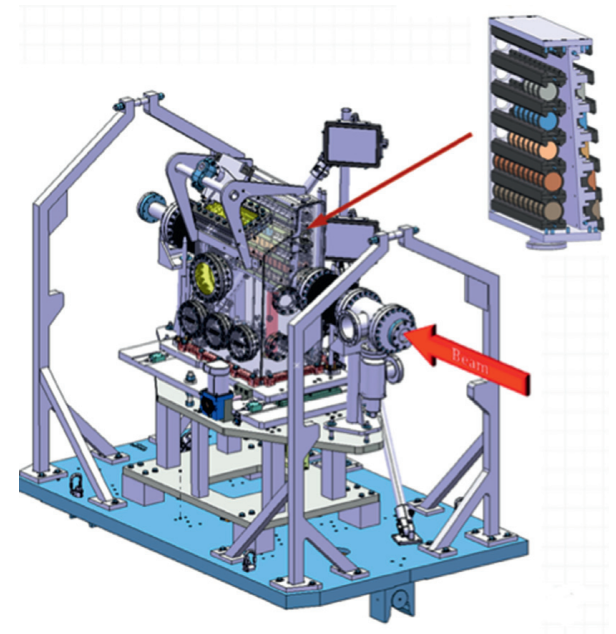

FIGURE 5: General assembly of the test-bench [15].

TABLE 3: Beam parameters, medium-intensity tests on Inermet ${ }^{\circledR} 180$.

\begin{tabular}{lcc}
\hline & Experiment parameters & Simulation parameters \\
\hline Proton energy & $440 \mathrm{GeV}$ & $440 \mathrm{GeV}$ \\
Number of bunches & 24 & 24 \\
Pulse intensity & $2.7 \mathrm{e} 12$ protons & $2.7 \mathrm{e} 12$ protons \\
Bunch spacing & $25 \mathrm{~ns}$ & $25 \mathrm{~ns}$ \\
Impact point & Center of specimen & Center of specimen \\
Beam transverse dimension & $1.4 \times 2 \mathrm{~mm}^{2}$ & $2.5 \times 2.5 \mathrm{~mm}^{2}$ \\
\hline
\end{tabular}

TABLE 4: Beam parameters, high-intensity tests on Inermet ${ }^{\circledR} 180$.

\begin{tabular}{lcc}
\hline & Experiment parameters & Simulation parameters \\
\hline Proton energy & $440 \mathrm{GeV}$ & $440 \mathrm{GeV}$ \\
Number of bunches & 72 & 60 \\
Pulse intensity & $9.05 \mathrm{e} 12$ protons & $9 \mathrm{e} 12$ protons \\
Bunch spacing & $25 \mathrm{~ns}$ & $25 \mathrm{~ns}$ \\
Impact point & $2 \mathrm{~mm}$ from the flat surface & $2 \mathrm{~mm}$ from the flat surface \\
Beam transverse dimension & $1.9 \times 1.9 \mathrm{~mm}^{2}$ & $2.5 \times 2.5 \mathrm{~mm}^{2}$ \\
\hline
\end{tabular}

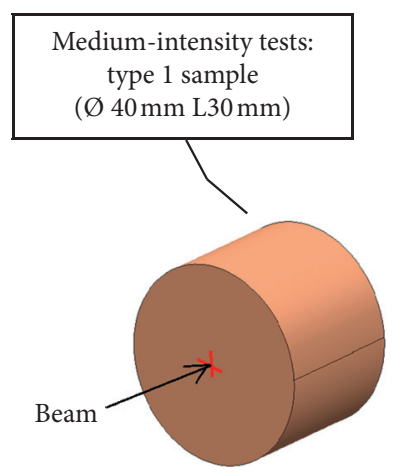

(a)

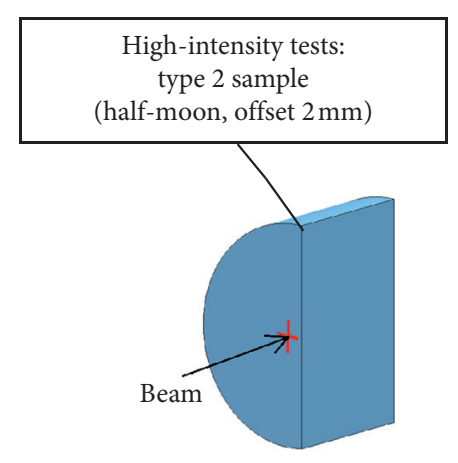

(b)

Figure 6: Material specimen shapes for medium intensity type 1 (a) and high intensity type 2 (b) [15]. 
The stainless steel vacuum vessel hosted a series of view ports to allow online measurements and postmortem observations. Each of these ports housed a radiation-hard window, mechanically designed to withstand internal vacuum and fragment impacts. The radiation-hard window can maintain its transparency in case of doses up to $8 \mathrm{MGy}$, three orders of magnitude higher than the dose expected for this experiment at the viewport location. One view port allowed the transmission of a laser beam for laser Doppler vibrometer measurements on type 1 specimens (lateral window). A second port was dedicated to the image acquisition of type 2 samples while exposed to high intensity shots. The vessel was equipped with beryllium windows at the beam entry and exit port, Figure 7.

1.4. Instrumentation Requirements. The whole instrumentation was designed in order to capture, in terms of amplitude and frequency, the expected signals. Extensive simulations were performed with the explicit finite element code Autodyn during the design phase on materials for which constitutive models were more reliable (Inermet ${ }^{\circledR} 180$ and Glidcop $\left.{ }^{\circledR} \mathrm{AL}-15\right)$ to define DAQ requirements. Figure 8 shows an example of the expected radial velocity on an Inermet ${ }^{\circledR} 180$ specimen. Requirements for the experiment are resumed in Table 5.

\section{Experiment Instrumentation}

Given the radiation level expected during the experiment (up to $25 \mathrm{kGy}$ to the embedded instrumentation [16]), only equipment devised for use under ionizing radiation could be installed in situ. All embedded components (strain gauges, thermal sensors, adhesives, connectors, and cables) had a resistance before failure higher than $250 \mathrm{kGy}$ [17]. The most delicate components, such as the vibrometer and the highspeed camera, were positioned inside a shielded bunker, $40 \mathrm{~m}$ upstream of the experimental area.

2.1. Strain Gauges. During the design phase, as discussed in Section 3.1, several types of strain gauges were characterized in order to define the correct choice for the HiRadMat environment and the specifications of the experiment. The solution adopted included HBM 1-XY91-3/120 strain gages bonded with Araldite Standard epoxy adhesive.

2.2. Thermal Probes. The temperature response of each material after the impact was monitored by PT100 thermal probes glued with LOCTITE $^{\circledR}$ STYCAST 2850 FT, a black, thermally conductive epoxy encapsulant.

2.3. Laser Doppler Vibrometer. The motion of an object can be measured evaluating the phase shift of a light beam. Laser Doppler vibrometry is a proven technique for this kind of measurement [18-20]. The main advantages of the technique are as follows: the structural behaviour of the structure is not altered by the mass of sensors, working distance between structure and instrument limited only by the source power and the maximum size of the optical system, punctual measurement (highly focused beam), and frequency response not limited by resonances.

2.3.1. Working Principles. The output laser of the vibrometer is composed by two beams. The first one is directed to the measured surface, and the second one is shifted in frequency and sent to the photodetector (reference beam); the frequency shift must be higher than the expected Doppler modulation of the surface. A part of the reflected light returns to the vibrometer and is combined with the reference beam; the interference between the reference and the reflected beam is produced on the photodetector. If the measured surface is moving, the phase or the frequency of the interference changes, producing a Doppler modulation of the interference on the photodetector. Direction and amplitude of the movement are given by such Doppler modulations.

The reference light wave impacting the photodetector is given by

$$
E_{R}(t)=A_{R} \exp \left(i\left(w_{l}+w_{c}\right) t\right) .
$$

The reflected light wave is given by

$$
E_{D}=A_{D} \exp \left(i\left(w_{l} t+\phi_{D}(t)\right)\right),
$$

where $A_{R}$ and $A_{D}$ are the amplitudes of the reference and reflected lights impacting the photodetector, $w_{l}$ is the radial frequency of the light wave impacting the surface under testing, and $w_{c}$ is the heterodyne radial frequency (shift of the reference beam).

The information of the surface movement is carried by the Doppler of the reflected light:

$$
\phi_{D}=\frac{4 \pi \cdot z(t)}{\lambda}
$$

where $z(t)$ is the time-dependent position of the surface.

The intensity of the light impacting the photodetector is the norm of the sum of both beam intensities:

$$
\begin{aligned}
I(t) & =\left|E_{R}(t)+E_{D}(t)\right|^{2}, \\
& =A_{R}^{2}+A_{D}^{2}+2 A_{R} A_{D} \cos \left(w_{C} t+\phi_{D}(t)\right) .
\end{aligned}
$$

A constant DC current is created by the terms $A_{R}^{2}$ and $A_{D}^{2}$, which represent the light intensity of the reference beam and the returning light: the DC current produces a unavoidable shot noise. The motion information is carried inside the phase modulation of the third term of equation (4). The conversion from light intensity to current is described in [21].

2.3.2. Adopted Solution: Vibrometer RSV-I-150 from Polytec $^{\circledR}$. A laser beam with $1500 \mathrm{~nm}$ wavelength was used to perform measurements in the near infrared, instead of the traditional green laser beam (wavelength $633 \mathrm{~nm}$ ). The shot noise produced by the light intensity of reference and returning beams, $A_{R}^{2}$ and $A_{D}^{2}$, is tuned to create a noise higher than all electronic noise; the detection is called shot 


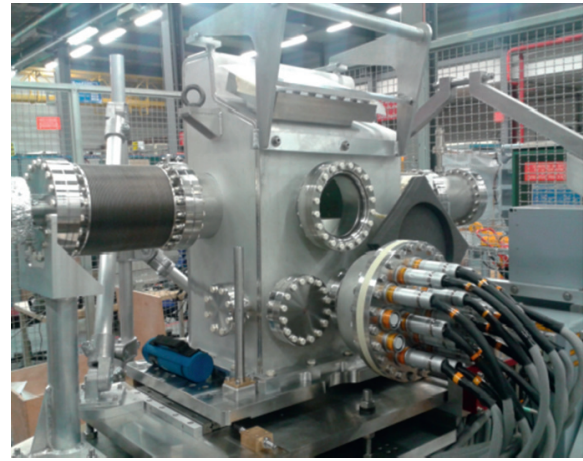

(a)

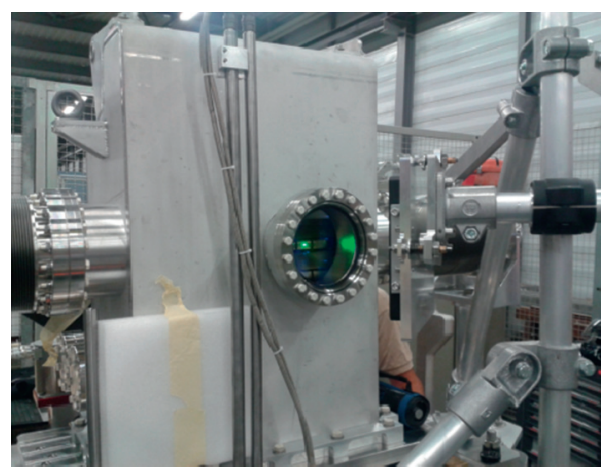

(b)

FIgURE 7: Assembled vacuum tank with DAQ cables and connectors (a); viewport for LDV measurements (b) [15].
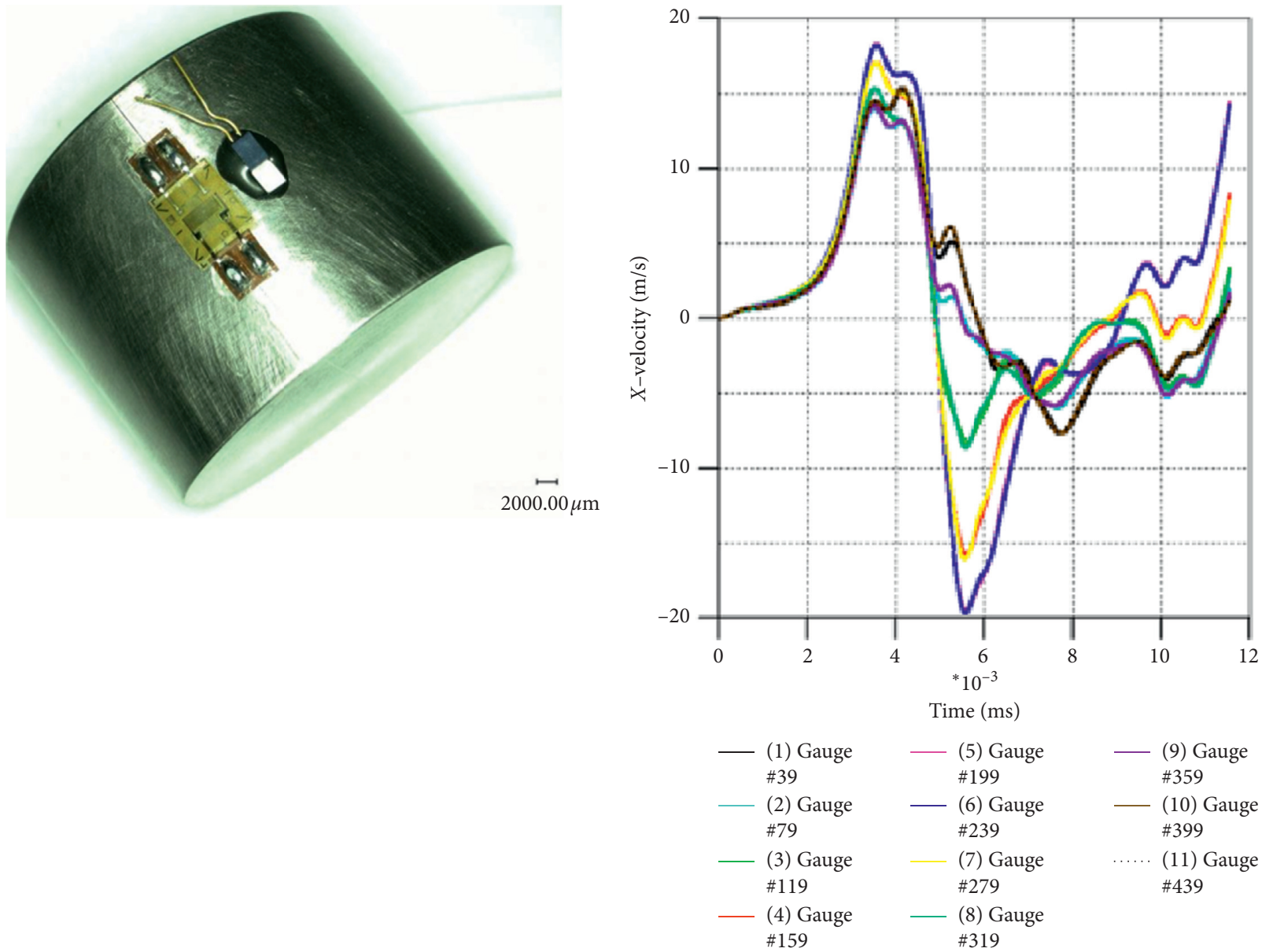
- (9) Gauge \#359 (10) Gauge \#399 (11) Gauge \#439

(a)

(b)

FIGURE 8: Inermet ${ }^{\circledR} 180$ specimen with Pt100 and bidirectional strain gauge (a); expected radial velocity on Inermet ${ }^{\circledR} 180$ sample, energy $440 \mathrm{GeV}, 3 \mathrm{e} 12$ protons (b) [15].

noise limited [18]. In this case, the reference light is much more intense than the returning Doppler shifted light: $A_{R}^{2} \gg A_{D}^{2}$ and the shot noise is dominated by the returning light. The carrier-to-noise (CNR) ratio is defined as the ratio of the received modulated signal power to the shot noise power from the reference beam: the higher the CNR, the better the quality of the signal.
The CNR is proportional to the wavelength and to the quantum efficiency of the detector $Q_{E}$; it is also independent of the light intensity of the reference beam. These characteristics are matched by telecom detectors, which present high $Q_{E}$ with a band of $1500 \mathrm{~nm}$. Changing the wavelength from $633 \mathrm{~nm}$ to $1550 \mathrm{~nm}$ increases the CNR by a factor of 2.4 while. According the DIN/IEC 60825 laser safety norm [22], 
TABLE 5: Instrumentation requirements.

\begin{tabular}{lcccccc}
\hline & $\begin{array}{c}\text { Type of } \\
\text { measurement }\end{array}$ & Max amplitude & $\begin{array}{c}\text { Time } \\
\text { response }(\mu \mathrm{s})\end{array}$ & Quantity & Sensor type & Sampling frequency \\
\hline \multirow{3}{*}{ Electrical systems } & Surface strain & $3000 \mu \mathrm{m} / \mathrm{m}$ & 1.5 & 244 & Strain gauges & $4 \mathrm{MHz}$ \\
& Temperature & $150^{\circ} \mathrm{C}$ & 1 & 36 & Pt100 & $100 \mathrm{~Hz}$ \\
Optical systems & Vacuum & $10^{-6} \mathrm{mbar}$ & 1 & 1 & Pirani gauge & $100 \mathrm{~Hz}$ \\
& Radial velocity & $24 \mathrm{~m} / \mathrm{s}$ & 1.5 & 1 & Laser Doppler vibrometer & $4 \mathrm{MHz}$ \\
& Particle front propagation & $316 \mathrm{~m} / \mathrm{s}$ & 5 & 1 & High-speed camera & $20 \mathrm{kfps}$ \\
\hline
\end{tabular}

the power of laser with a wavelength above $1400 \mathrm{~nm}$ can reach $10 \mathrm{~mW}$, ten times higher than a visible laser with a wavelength of $633 \mathrm{~nm}$ which is limited to $1 \mathrm{~mW}$, thus improving the CNR of $10 \mathrm{~dB}$, Figure 9.

2.4. System for Image Acquisition. In those experiments where it was to be foreseen sample explosions due to the impact of high intensity beams, a specific system was developed making use of a high-speed camera, flash lamps, or led lights to provide the light during acquisition and mirrors to create the optical path from the test stand to the protected bunker in which the camera was placed.

2.4.1. High-Speed Camera. The video camera used during the experiment is a Redlake MotionXTra HG-100K, with a working frequency of $1 \mathrm{kHz}$ at an $800 \times 600$ px resolution; the frame rate can be further increased to a maximum of $100 \mathrm{kHz}$ by reducing the resolution down to $32 \times 24$ pixels.

The image acquisition can be triggered manually or by an external transistor-transistor logic trigger signal (TTL): a cyclic image storage system on on-board memory permits to keep in memory up to $2 \mathrm{~GB}$ of data, saving a chosen number of images before the trigger signal advent: the number of images and the maximum frame rate is governed by the imposed resolution, while the electronic shutter is synchronized with the internal camera clock and can be reduced down to $5 \mu \mathrm{s}$.

The system consists of Nikkor $1000 \mathrm{~mm}$-equivalent catadioptric lens, a mirror system composed by three mirrors and relative adjustable holders, and a LAN-connected PC for remote controlling of the camera.

In order to conveniently set the video camera resolution, frame rate, and shutter time, the following requirements were imposed:

(i) Reduction of the blur effect given by the displacement of the exploded particle front during the acquisition of each frame

(ii) Sufficiently high number of pixels on the observable area through the window $\left(100 \times 100 \mathrm{~mm}^{2}\right)$

(iii) Acquisition of at least 5 pictures of the phenomenon (duration of the explosion- $400 \mu \mathrm{s}$ )

The blur effect could be reduced only by decreasing the shutter time down to the minimum of $5 \mu \mathrm{s}$ : this gives a displacement during one frame of about $1.25 \mathrm{~mm}$; each pixel must therefore be smaller than $0.625 \mathrm{~mm}$. The best

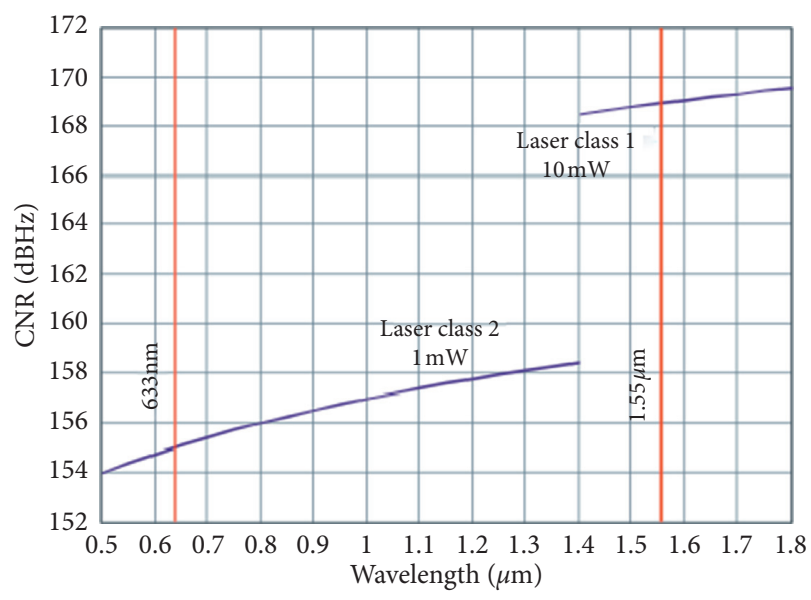

FIGURE 9: Theoretical carrier-to-noise ratio drawn over wavelength, with the measurement light power allowed for class 2 lasers for wavelengths up to $1.4 \mu \mathrm{m}$ and class 1 lasers for wavelengths above $1.4 \mu \mathrm{m}$ [18].

compromise between resolution and frequency was found with $224 \times 224$ pixels at $20 \mathrm{kHz}$ (one picture every $50 \mu \mathrm{s}$ ).

These settings require very intense and synchronized lighting during the acquisition; in addition, the high radiation level around the tank does not allow to embark sensitive electronic devices such as LED lamps or modern photographic flashes [23]. For these reasons, xenon flash lamps were chosen.

2.4.2. Lighting System. A xenon flash lamp (XFL) is capable to produce a significant amount of light by giving an electrical surcharge to a tube filled with xenon gas: for example, a small, standard photographic flash with 32 GN (Guide Number) can easily reach 50000 lumens, while an LED flash hardly can get 2-5000 lumens. Anyway, a modern xenon flash lamp has a motherboard embarking several $\mu$-electronic devices with low radiation resistance: the adopted solution is the use of old flashes ( 1970 s-80 s) such as the proposed model Panasonic PE-145. These flashes rely entirely on condensers, diodes, and inductive spires, highly resistant to radiations. See a standard flash circuit in Figure 10.

The most important issue of this system is the lighting duration and synchronization, since a standard xenon flash lasts few hundreds of microseconds with a Gaussian-like light output time curve: Figure 11 shows the typical discharge curve of a flash condenser, assuming that the light output is proportional to the current passing from the capacitor to the xenon tube. The useful lighting time is 


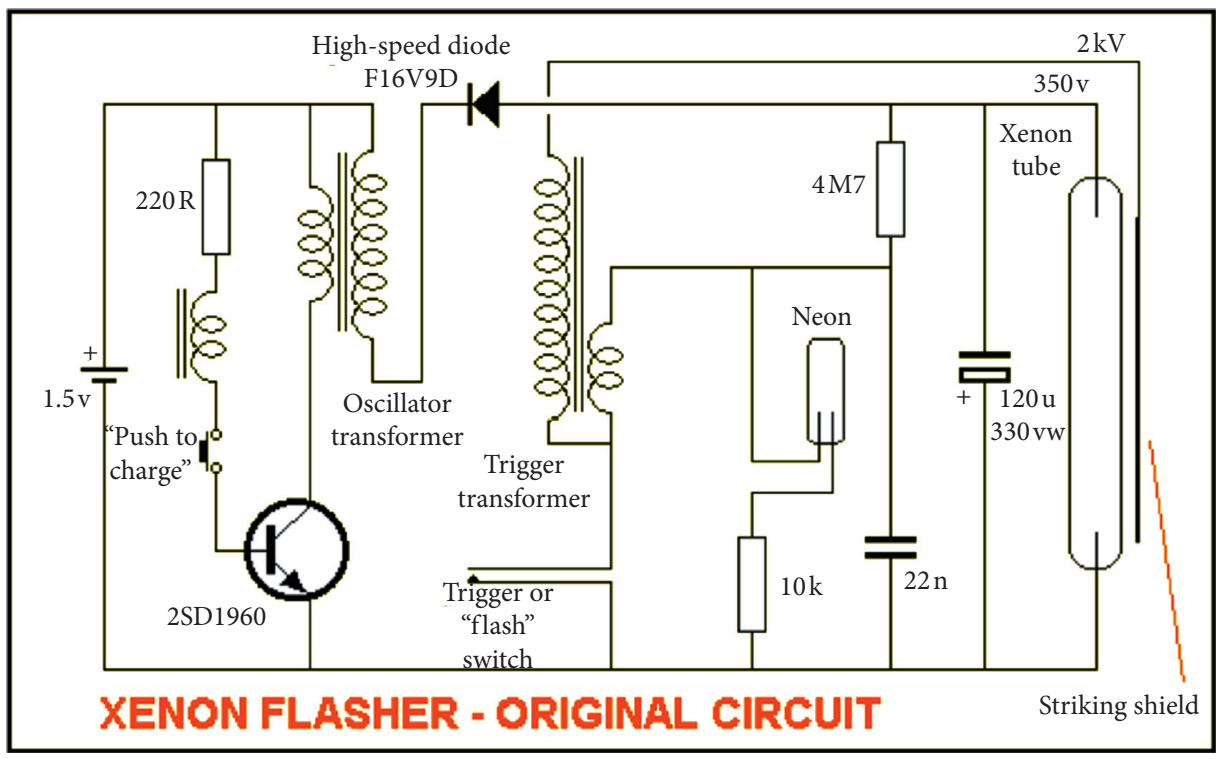

FIgURE 10: Example of xenon flash circuit (Polytec).

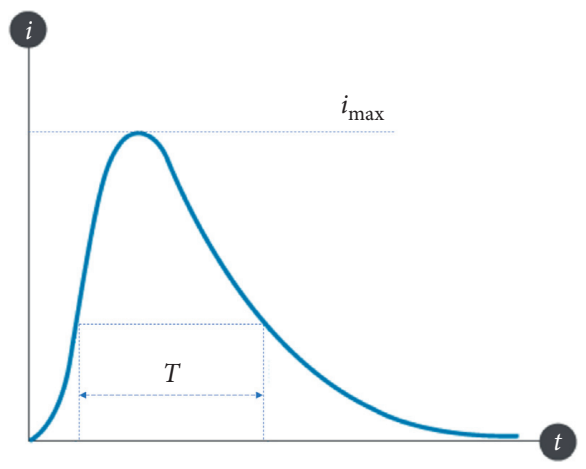

Figure 11: Characteristic time evolution of the current inside the xenon tube. Time constant $\mathrm{T}$ is in the order of $100-200 \mu$ s for standard flashes.

indicated by the time constant $T$ which usually is in the order of few hundreds of microseconds.

Since the whole phenomenon lasts about $500 \mu \mathrm{s}$, highcapacity condensers were adopted to increase the time constant of each flash. On the contrary, increasing the output power of the flash was not a viable option, because the light intensity at the peak (corresponding to $i_{\max }$ in Figure 11) could overexpose the video camera sensor, returning a blank image.

By default, PE-145 flashes are equipped with a capacitor featuring $350 \mathrm{~V}$ maximum voltage and $250 \mu \mathrm{F}$ capacitance, for a total useful lighting time of $150 \mu \mathrm{s}$ : increasing the capacitance of the condenser to $3300 \mu \mathrm{F}$ allows to increment the total lighting time to about $1 \mathrm{~ms}$. Such condensers can store high amounts of energy and need a remote safe discharge system.

\section{Before the Experiment: Tests on the Instrumentation}

Instrumentation devices described in Section 2 were guaranteed by the suppliers over a certain range of amplitude, resolution, time response, and frequency.
However, the most energetic impacts were expected to require instrumentation performances higher than what specified in the documentation. These devices were therefore tested before the experiment, generating, in ad hoc testing samples, shockwaves similar to those simulated for HiRadMat specimens.

3.1. Qualification Test on the Strain Gauges. Suppliers certified the dynamic response of proposed strain gauges up to $50 \mathrm{kHz}$; tests using Hopkinson Bar [24] tests were, therefore, performed in Politecnico di Torino in order to check the dynamic response of strain gauges and glue, to evaluate the signal-to-noise ratio, the accuracy of the measurements, and the maximum measurable amplitude.

During tests performed at Politecnico di Torino, Figure 12 , strains above $5000(\mu \mathrm{m} / \mathrm{m})$ were reached, with a strain rate higher than $1 \times 10^{3} \mathrm{~s}^{-1}$.

The signal acquisition was performed by a Gen2i from $\mathrm{HBM}^{\circledR}$ with a Uni1M ISO card. Strain gauges from HBM were adopted (model: 1 - LY11 - (3/350)) with a resistance of $350 \Omega$ in a quarter bridge configuration. Wheatstone bridge completion was composed of 3 thin film resistors $(350 \Omega)$. BNC cables and $33 \mathrm{~m}$ of double shielded cables were used to connect the Wheatstone bridge to the data acquisition system.

A first test was performed on a stainless steel sample, Figure 13, with the objective to check the time response of the strain gauges (both resistive and semiconductor types were tested). A strain of $4000(\mu \mathrm{m} / \mathrm{m})$ in $4 \mu$ s was recorded; the bandwidth of the phenomenon was $200 \mathrm{kHz}$. The amplitude difference between resistive and semiconductor strain gauges was introduced by a bending moment on the sample (resistive and semiconductor strain gauges were placed with an angular spacing of $180^{\circ}$ between each one, in quarter bridge configuration). 


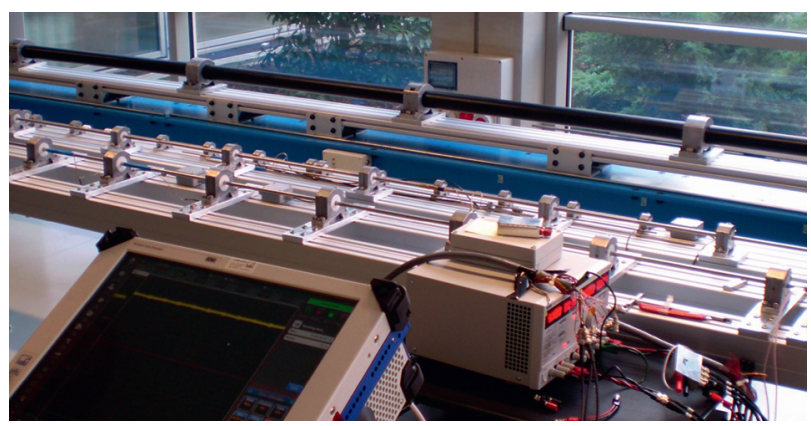

Figure 12: Hopkinson bar test-bench at Politecnico di Torino.

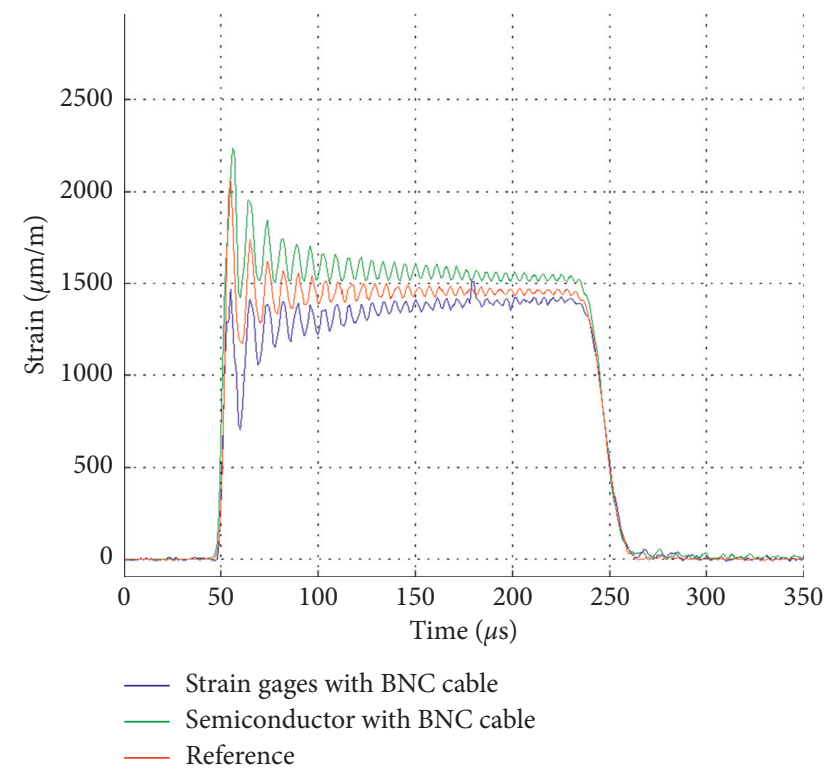

FIGURE 13: SPHB measurement on stainless steel sample, reference taken in the input bar, and acquisition via resistive and semiconductor strain gauges.

A second test was performed with an aluminium sample, with the objective to measure strains above $3000(\mu \mathrm{m} / \mathrm{m})$ and to verify the effectiveness if the glue at such deformations. A strain of $5500(\mu \mathrm{m} / \mathrm{m})$ was obtained in $4 \mu \mathrm{s}$. All gauges were still glued on the sample even after the severe plastic deformation generated.

Validation tests on the strain gauges are summarized in Table 6. Semiconductor strain gauges were discarded due to their high sensitivity to radiation and because of the calibration needed before each experiment, the nonlinear behaviour, and the high thermal sensitivity. In order to avoid signal distortion, the impedance of strain gauges, cables, and data acquisition system must match. Strain gauges from HBM $^{\circledR}$ were selected (model: $1-\mathrm{XY91}-(3 / 120)$ ) with a resistance of $120 \Omega$ in a quarter bridge configuration. The strain gauges measure in two directions with $90^{\circ}$ of difference.

During the in-beam tests, a perturbation in the strain gages signal was observed during the first $20 \mu$ s after the passage of the beam, as can be seen in Section 6.1. This perturbation is induced by the coupling of the strain gage circuit to the electromagnetic fields as generated by the particle beam, as discussed in [25]. This perturbation makes the data taken during this time-slot largely unusable. Being this blackout an undesirable effect, in the case of the experiments under discussion it did not impede a correct exploitation of the data either thanks to the complementarity of the strain gages with other measurement techniques not affected by this perturbation as the LDV, or because all useful information was mostly outside of the initial $20 \mu$ s.

3.2. Qualification Test on the Laser Doppler Vibrometer. The vibrometer was provided by Polytec ${ }^{\circledR}$; a standard RSV-I150 was tuned to match the required speed amplitude of $24 \mathrm{~m} / \mathrm{s}$ and the bandwidth of $2.5 \mathrm{MHz}$. Amplitude, bandwidth, frequency response, resolution, signal delay, and reflectivity of the samples were determined with ad hoc tests at CERN. A shaker from MB Dynamics ${ }^{\circledR}$ model MB Dynamics Modal 50A with a signal generator from Agilent ${ }^{\circledR}$ model Agilent 33210A was adopted for the test.

The vibrometer was directed towards a first mirror; the laser beam was then reflected to a second mirror, at a distance of $40 \mathrm{~m}$, and directed to the sample, positioned behind a vacuum window. The sample was glued on the shaker, Figure 14; the vacuum window was compatible with the wavelength of the two lasers $(632 \mathrm{~nm}$ for the measuring laser beam, $1550 \mathrm{~nm}$ for the beamer). The shaker was excited by a sine sweep from $5 \mathrm{~Hz}$ to $1 \mathrm{kHz}$.

The test was successful, showing a good matching between generated and acquired signals, Figure 15 and Table 7.

3.3. Qualification Test on the Acquisition Cables. The signals expected were around $8(\mathrm{mV} / \mathrm{V})$, with the data acquisition $40 \mathrm{~m}$ away from the test zone. To verify the possible distortion or attenuation of the acquired signal in the cables, specific tests were performed with an Agilent ${ }^{\circledR}$ 4395A Network/Spectrum/Impedance analyser, increasing the frequency from 1 to $2000 \mathrm{kHz}$.

According the simulations, the characteristic frequencies of the physical phenomenon were below $200 \mathrm{kHz}$ : no decrease of magnitude and no significant phase shift can be seen in this area, Figure 16.

\section{Data Acquisition System}

The acquisition system was designed to record data from the vibrometer and strain gauges with a bandwidth of $2(\mathrm{MS} / \mathrm{s})$, thus requiring a data acquisition board with a frequency of $4 \mathrm{MHz}$.

4.1. PXIe. The system is build using a PXI express chassis (PXIe-1075) with a PXI express controller (PXIe-8115) running LabVIEW in real time on the PharLap operating system. It featured 12 data acquisition cards (PXIe-6124), each having 4 simultaneously sampled analogic inputs at $4 \mathrm{MS} / \mathrm{s}$ per channel with 16 bits of resolution.

A card dedicated to the temperature data acquisition was also included (PXIe-4357), capable to record up to 20 
TABLE 6: SPHB qualification test of strain gauges.

\begin{tabular}{lcc}
\hline & Resistive strain gauges & Semiconductor strain gauges \\
\hline Signal & $8 \mathrm{mV}$ for $1500(\mu \mathrm{m} / \mathrm{m})$ & $400 \mathrm{mV} \mathrm{for} 1500(\mu \mathrm{m} / \mathrm{m})$ \\
Bandwith & Tested up to $200 \mathrm{kHz}$ & Tested up to $200 \mathrm{kHz}$ \\
Noise & $60(\mu \mathrm{m} / \mathrm{m})$ & $20(\mu \mathrm{m} / \mathrm{m})$ \\
Linear behaviour & Yes & No \\
Radiation hardness & As a wire & High sensitivity \\
Single event hardness & As a wire & SC doping \\
Calibration & Not necessary & Before every test \\
Amplifier & Not necessary & Not necessary \\
Impedance matching & Necessary & Necessary \\
\hline
\end{tabular}

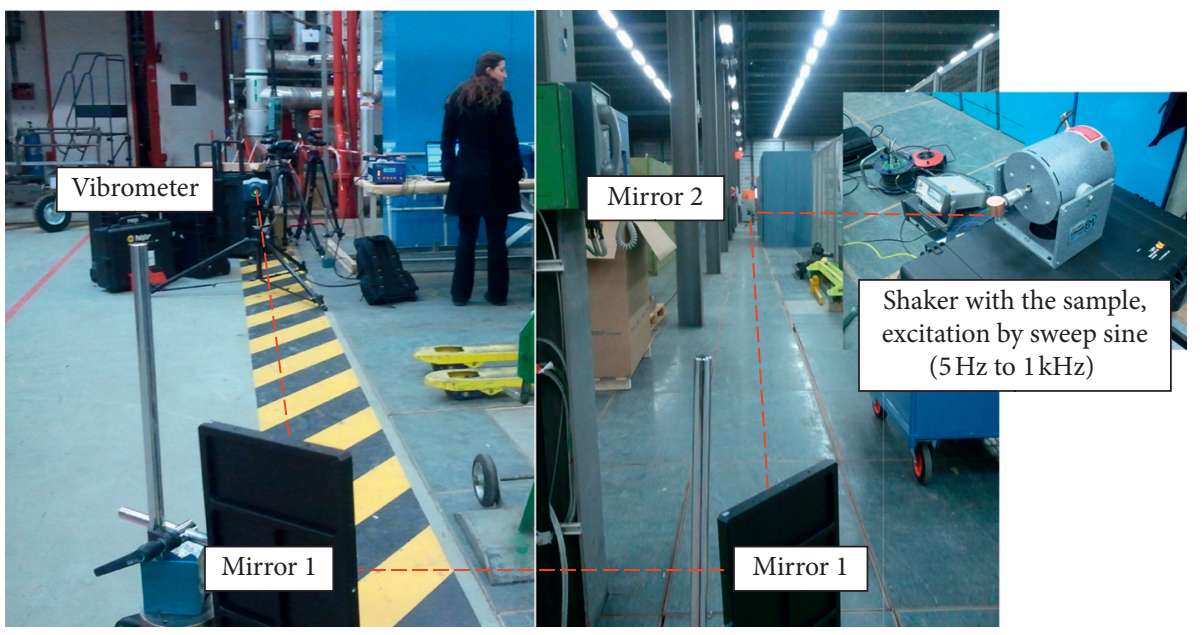

Figure 14: Qualification test for the vibrometer RSV-150 from Polytec ${ }^{\circledR}$. Mirrors 1 and 2 were provided by Polytec ${ }^{\circledR}$ with a size of, respectively, $420 \times 297 \mathrm{~mm}^{2}$ and $297 \times 210 \mathrm{~mm}^{2}$.

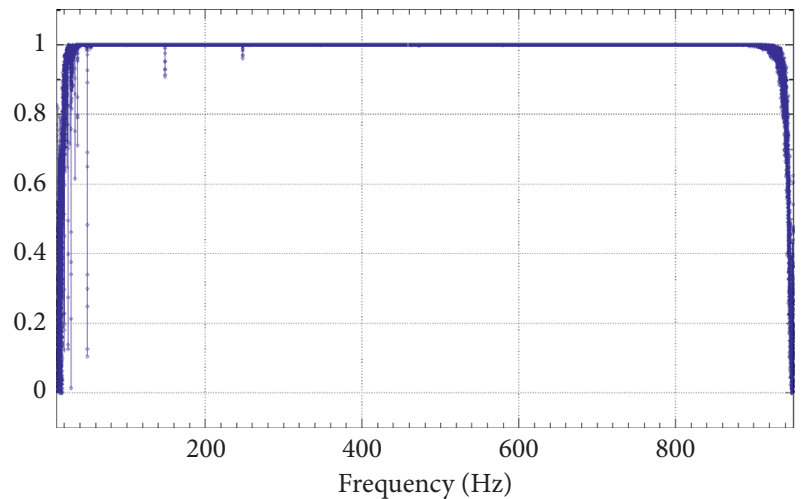

FIGURE 15: Generated and LDV-acquired signals are superposed $(5 \div 1000 \mathrm{~Hz})$

TABLe 7: Qualification tests on the LDV.

\begin{tabular}{lc}
\hline Reflectivity of HiRadMat samples & Acceptable \\
\hline $\begin{array}{l}\text { Measurement at } 40 \mathrm{~m} \text { with two mirrors and vacuum } \\
\text { window }\end{array}$ & Achieved \\
Sensitivity to external vibrations & Negligible \\
Sensitivity to mirrors stability and positioning & High \\
\hline
\end{tabular}

channels from the PT100 probes at $100(\mathrm{~S} / \mathrm{s} / \mathrm{ch})$. Another card monitored the different voltages from the power supplies (PXI-6289). A remote computer, referred to as the host computer, was used to configure the real-time system and receive the data online. As a safety measure, a remote reset has been foreseen to enable the power off and power on of the entire system in case of major issue, Figure 17.

The system recorded data from the strain gauges and the vibrometer at the maximum frequency during the first $100 \mathrm{~ms}$. After this period, the acquisition frequency was then decreased to $100 \mathrm{~Hz}$ to study effects and propagation of lower-frequency waves over a time of $30 \mathrm{~s}$. In addition, the system acquired voltages from the power supply and temperature signals from the PT100 probes at $100 \mathrm{~Hz}$ for $30 \mathrm{~s}$.

When the beam reached the facility, a trigger was generated and sent to the data acquisition system. The trigger signal was a TTL having a jitter of $\pm 1 \mathrm{~ms}$ : for this reason, the fast acquisition was performed using a pretrigger of at least $1 \mathrm{~ms}$ to avoid losing data. The slow acquisition at $100 \mathrm{~Hz}$ could start immediately after the trigger. In a posttriggered acquisition, the hardware starts the $\mathrm{A} / \mathrm{D}$ conversions after the trigger is received; the trigger signal in this case is referred to as the start trigger. In a pretriggered acquisition, the hardware starts acquiring data before the trigger signal is received. With this type of acquisition, the user can view the signal before the trigger event. In such applications, the hardware initiates data acquisition and stores the data in a circular buffer in the card memory. The buffer was large enough to ensure that the required number of pretrigger samples was stored; when it was full, it simply wrapped around and stored each subsequent sample over the 


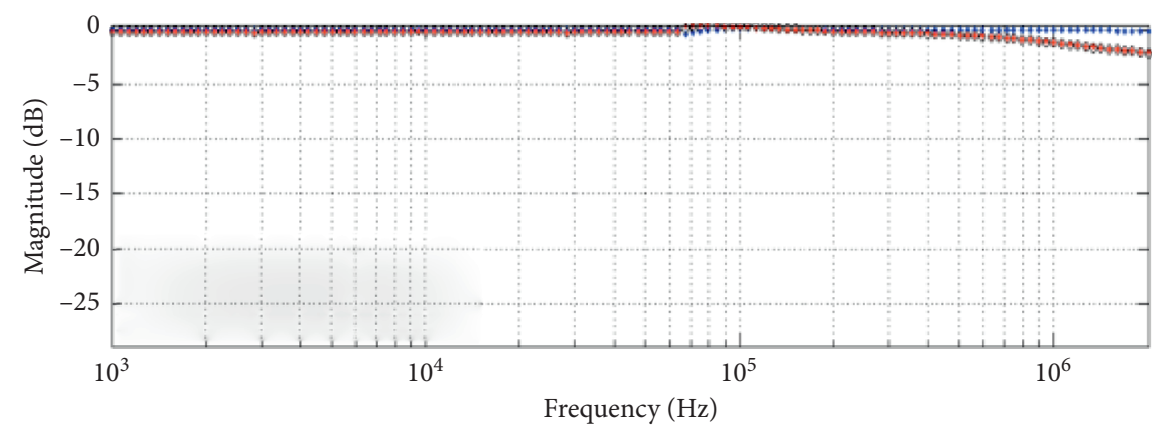

- Magnitude cable bunker $(10(\mathrm{~m}))$

- Magnitude cable table (45 (m))

$\diamond$ Combination of both transfer functions

(a)

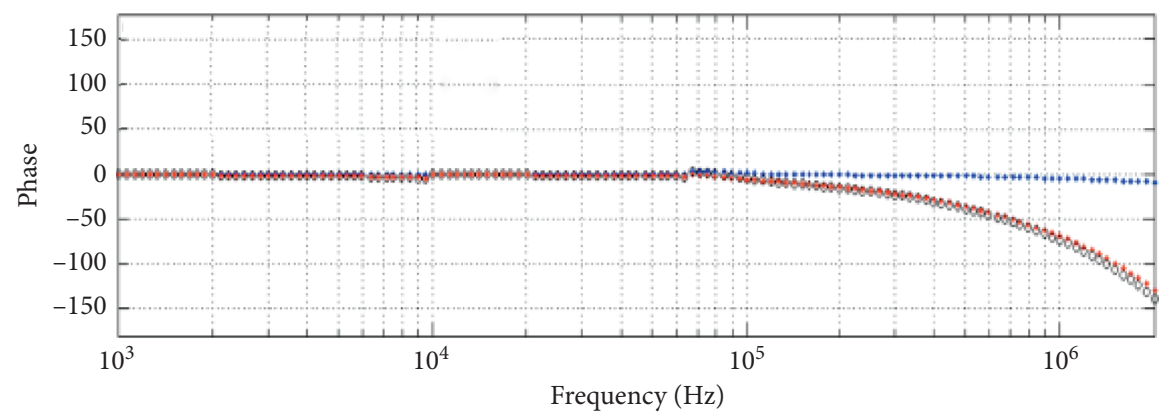

- Phase cable bunker $(10(\mathrm{~m}))$

- Phase cable table (45 (m))

$\diamond$ Combination of both transfer functions

(b)

Figure 16: Test on the instrumentation cables.

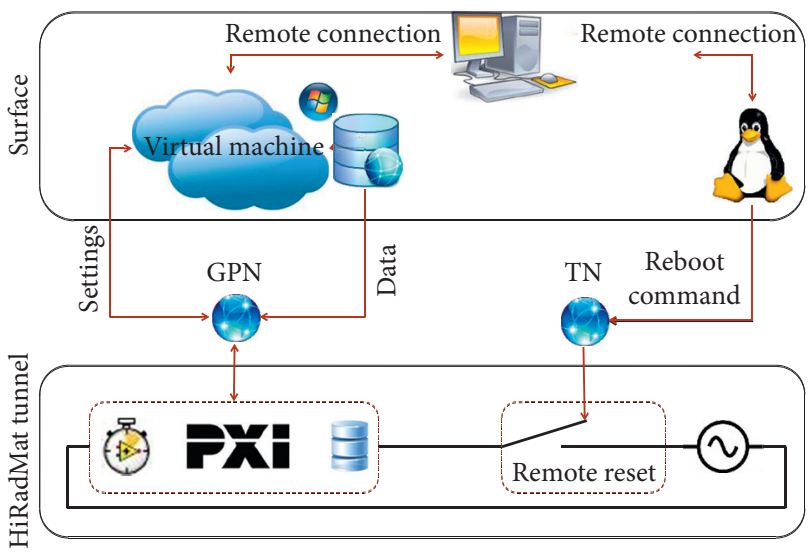

Figure 17: SHC-DAQ architecture.

oldest sample in memory. The primary responsibility of the trigger mechanism was to stop the acquisition so that the samples left in memory represented the slice-in-time the user wanted. The trigger signal in this case is referred to as the reference trigger.

4.2. Software. The software structure running on the realtime target was made using three parallel loops, Figure 18. The first loop was dedicated to the communications with the host computer.
The communication was handled over the network using Shared Variables from National Instrument. The configuration could be viewed on the host computer, changed and sent to the target; the configuration actually used on the PXI was then sent back from the real-time environment to the host. As soon as a new configuration was received by the PXI, the second loop, assigned to the hardware access, was activated. The configuration was applied on the three card types in parallel. The 12 PXIe6124 cards, dedicated to strain gauges and vibrometer, were armed using the reference trigger method to allow visualizing a couple of milliseconds before the trigger. The PXIe-4357 and PXI-6289, dedicated, respectively, to PT100 and voltages, are armed in two parallel tasks using the start trigger method. When the trigger was received, data were pushed to the third loop, dedicated to data handling. Their data were saved locally on the hard disk of the PXI system and published on the network using Shared Variables. Thus, the connected host computer was receiving the data shortly after the trigger.

As soon as the PXIe-6124 running at 4 (MS/s) using reference trigger had finished its task, the cards had to be reconfigured to record data at $100 \mathrm{~Hz}$ for $30 \mathrm{~s}$. This reconfiguration of the hardware resulted in a dead band where no data can be acquired using these cards. This time could be up to $1 \mathrm{~s}$, Figure 19. 


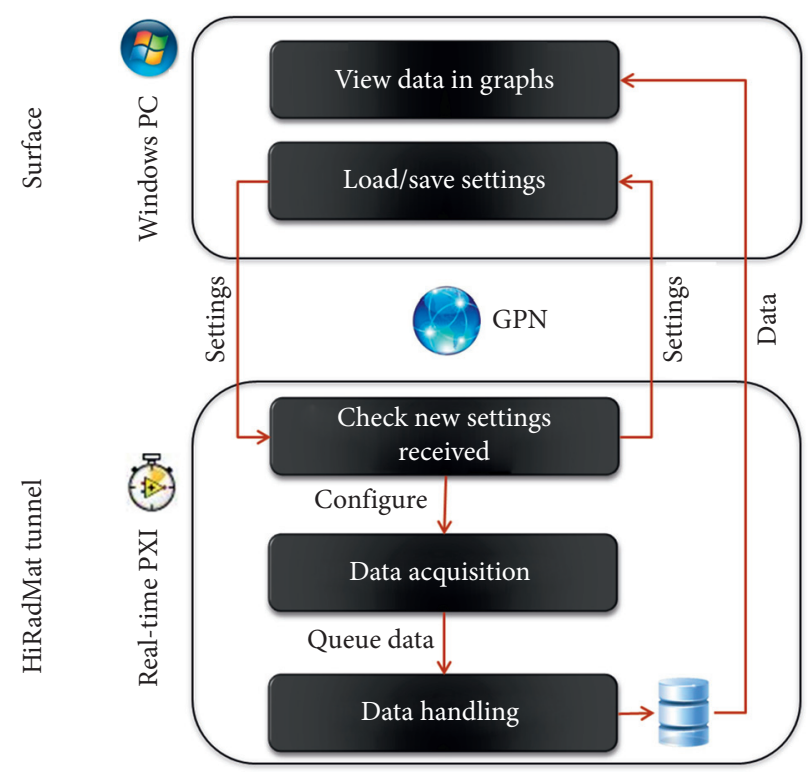

FIGURE 18: Communication structure.

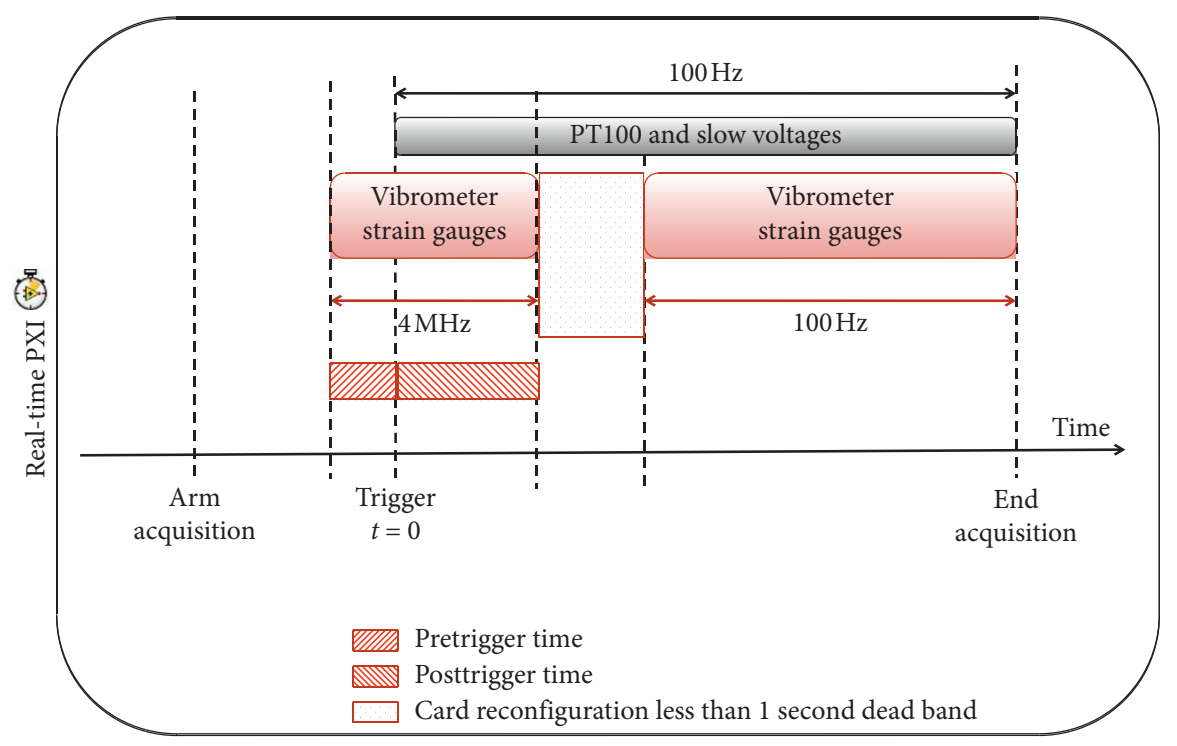

Figure 19: Data acquisition scheme.

When the data were stored locally, the user could download the data to his computer for offline analysis. During this time, the data acquisition system is automatically reconfigured waiting for another trigger to occur. The user can still change the configuration if needed. The system will be automatically paused and rearmed with the new settings.

During the entire experiment in HiRadMat, the application never failed to read a trigger and save the data. In a single occasion, the remote reset had to be used, because the real-time system was not answering to any command; in this particular situation, a single event has been suspected as the system restarted very easily following the power cycle.

4.3. Signal Cabling and Multiplexing Switch. In total, 244 strain gauges and $36 \mathrm{Pt} 100$ probes were installed, requiring a total of 1088 wires. The facility had signal wires installed from the experimental area to a patched panel in the bunker, but only 144 lines were available. In order to reduce the number of signal lines, a switch was designed and installed in the test table. In addition to the signal cables, one power cable for operation of the motors was available.

The design of the switch considered three constraints: radiation resistance, minimum signal distortion, and fail safe remote operation. Solid-state relays were discarded due to the dose rate. After a market research, no commercial device fulfilled the requirements, especially the radiation tolerance; a custom printed circuit board (pcb) design was therefore produced, Figure 20. For communication applications, electromechanical $24 \mathrm{~V}$ relays were chosen. The topology used was a tree solution, with four layers of switches to equalize the electrical path of all channels and a multiplexer rate of $8: 1$. The activation of the 


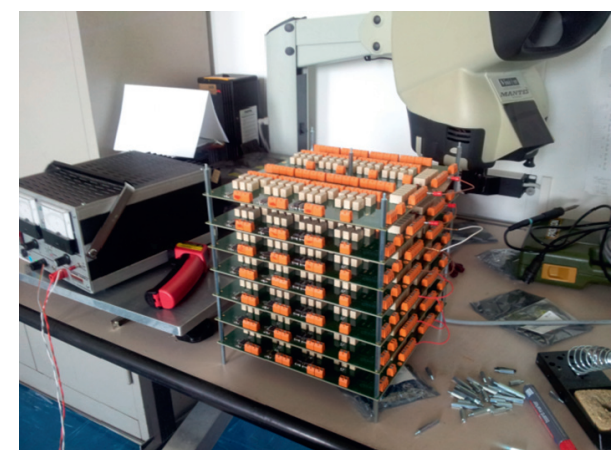

FIgURe 20: Pcbs cards used during the experiment.

relay coils was also implemented with other relays controlled directly from the bunker using the power cable. In this way, all the available signal lines could be used to test each sample line sequentially. The resistors to complete all Wheatstone bridges were installed in the switch pcbs, as they would not fit inside the vacuum tank. Due to the relatively big size of the relays, the final solution consisted of 7 pcbs of $235 \times 250 \mathrm{~mm}^{2}$ with one of the boards acting as master. The boards were contained in two aluminium boxes on the experiment table, with forced ventilation to dissipate the switching heat. Before the test, the switch was exhaustively tested, including extreme cases without ventilation and high activity.

The facility signal cables were also a limiting factor in terms of signal distortion. A study was carried out to obtain their equivalent transmission line parameters (intrinsic impedance $z_{0}$ and $\gamma$ ). An intrinsic impedance $z_{0}$ of approximately $100 \Omega$ was obtained for the relevant bandwidth. To minimize reflections, $120 \Omega$ gauges were used instead of the more common ones with a resistance of $350 \Omega$. Also, in all the cabling for the gauges and the switch, $120 \Omega$ shielded twisted pair was chosen. However, in the instrumentation side, it was decided not to adapt the input impedance, using a National Instruments card with $1 M \Omega$ in parallel with $20 \mathrm{pF}$. Finally, one signal pair per sample line was equipped with an adapted radiation-qualified differential amplifier. These lines were connected both before and after amplification, in order to have an estimation of the quality of the signals measured. Due to an increment in the number of measurement points required, this feature could not be used in the final test.

4.4. Power Supply. Three power supply voltages were required for the setup:

(i) $24 \mathrm{~V}$ for the operation of the switch relays

(ii) $9 \mathrm{~V}$ for the supply of the strain gauges

(iii) $5 \mathrm{~V}$ for the radiation-resistant differential amplifiers

Due to the high radiation expected in the experimental area (up to $25 \mathrm{kGy}$ ), the supplies too were located inside the bunker. The cables used were shielded to limit the interference. All power supplies used were linear and had a power ripple lower than $3 \mathrm{mV}$.

\section{Methods to Treat the Acquired Signal}

A huge quantity of data was acquired during the experiment in the HiRadMat facility. Some methods were developed in order to treat the acquired signals, distinguish between physical data and noise.

5.1. Smoothing Function. A locally weighted linear regression is used to smooth data acquired during the experiment; each smoothed value can be determined from neighbouring data points, within a span of 16 points. A weight function is defined by the data points contained within the span [26].

The regression weight function used is given by equation (5); $x$ is the predictor associated to the value to be smoothed, $x_{i}$ are the nearest neighbours of $x$ defined by the span, and $d(x)$ is the distance along the abscissa from $x$ to the most distant predictor. This weight function is characterized by the fact that the data point to be smoothed has the largest weight and the points outside the span have no weight. The smoothed value is given by a weighted linear least-square regression curve with a quadratic polynomial:

$$
w_{i}=\left(1-\left|\frac{x-x_{i}}{\mathrm{~d}(x)}\right|^{3}\right)^{3} \text {. }
$$

\section{Results}

In this section, results obtained on Glidcop ${ }^{\circledR}$ and Inermet ${ }^{\circledR}$ 180 are introduced. As this paper focuses on the developed instrumentation systems, no data analysis will be provided, expect for the smoothing function applied to reduce the noise on the signals and have a clear view on the strain measurement.

For medium intensity cylindrical samples, measurements were acquired in 4 points, with a $90^{\circ}$ hoop spacing between each one; three measurements were acquired by the strain gauges and one by the laser Doppler vibrometer. In the following figures, the three strain gauges are referred to as TOP, MIDDLE, and BOTTOM. Details of simulation and benchmarking methods are discussed in the document [27].

6.1. Medium-Intensity Test on Glidcop ${ }^{\circledR}$. The characteristics of the beam for the test and the simulation are listed in Table 8 . Note that there is a difference in beam dimension at the impact between what was requested and simulated, and what could be guaranteed during the experiment. The impact of this difference on the acquired results with respect to those simulated is expected to be small: in fact, strain gauges were placed at a distance of $20 \mathrm{~mm}$ from the beam axis. While the peak stresses and temperatures at the impact location will be higher in case of a smaller beam cross section, the amplitude of the cylindrical shockwave decays with the square root of the radial coordinate. At a distance of $20 \mathrm{~mm}$ from the beam axis, in the initial studies for the experiment, it was found that acquired strains on the external surface were very similar when using a beam with a dimension of $0.25 \times 0.25 \mathrm{~mm}^{2}$ vs. $2.5 \times 2.5 \mathrm{~mm}^{2}$ (of course 
TABle 8: Beam parameters, medium-intensity tests on Glidcop ${ }^{\circledR}$.

\begin{tabular}{lcc}
\hline & Experiment parameters & Simulation parameters \\
\hline Proton energy & $440 \mathrm{GeV}$ & $440 \mathrm{GeV}$ \\
Number of bunches & 72 & 72 \\
Pulse intensity & $4.66 \mathrm{e} 12$ protons & $4.66 \mathrm{e} 12$ protons \\
Bunch spacing & $25 \mathrm{~ns}$ & $25 \mathrm{~ns}$ \\
Impact point & Center of specimen & Center of specimen \\
Beam transverse dimension & $1.3 \times 1.3 \mathrm{~mm}^{2}$ & $2.5 \times 2.5 \mathrm{~mm}^{2}$ \\
\hline
\end{tabular}

with the same intensity and, therefore, the same stored energy). Obviously, since the purpose of medium-intensity tests was to limit peak temperatures and stresses at the center of the sample, minimizing the risks of melting/fragmentation, a larger beam size was preferred.

The raw data are presented and compared with the results obtained through Autodyn simulations. The signal measured by gauges is visible; still, it is difficult to distinguish each peak of the shock waves, Figure 4.

Applying the smoothing function, the first peak of the induced shockwave is still hidden in the perturbation of the beam, Figure 21. It disappears after about $20 \mu \mathrm{s}$, and the waves are visible and coherent with the strain model, in amplitude and shape.

The amplitude of the second peak is reduced by $500(\mu \mathrm{m} / \mathrm{m})$ between the strain model and the measurement.

The radial velocity, captured by the vibrometer, is compared with the simulated behaviour in Figure 22. Since the laser used for the measurement is not affected by the passage of the beam, no smoothing function is used on the signal. By contrast with the strain gauges signal, the first peak is clearly visible; the shape and the amplitude measured are in good agreement with Autodyn results.

6.2. Medium-Intensity Test on Inermet ${ }^{\circledR}$ 180. The characteristics of the beam adopted for the test and the numerical simulation are listed in Table 3.

Also in this case, the strong electromagnetic noise induced by the particle beam perturbed the strain gauge measurements during about $20 \mu \mathrm{s}$ after the impact, concealing the first deformation peak; however, this interference died out immediately after, allowing to capture the remainder of the phenomenon, Figure 23. The introduction of the smoothing function allows to assess the good agreement between simulations and the measurements, Figures 24 and 25.

6.3. High-Intensity Test on Inermet ${ }^{\circledR} 180$. The high-speed camera and the flash system allowed, for the first time, to the best of the authors' knowledge, to record images of the impact of a hadron beam on solid targets and of the effects this induced. The most remarkable phenomena occurred during beam impact on Inermet ${ }^{\circledR} 180$, the material with the highest stopping power [28].

The characteristics of the beam during this test are listed in Table 4. Also here, a slight difference is present in terms of beam transverse dimension experimentally

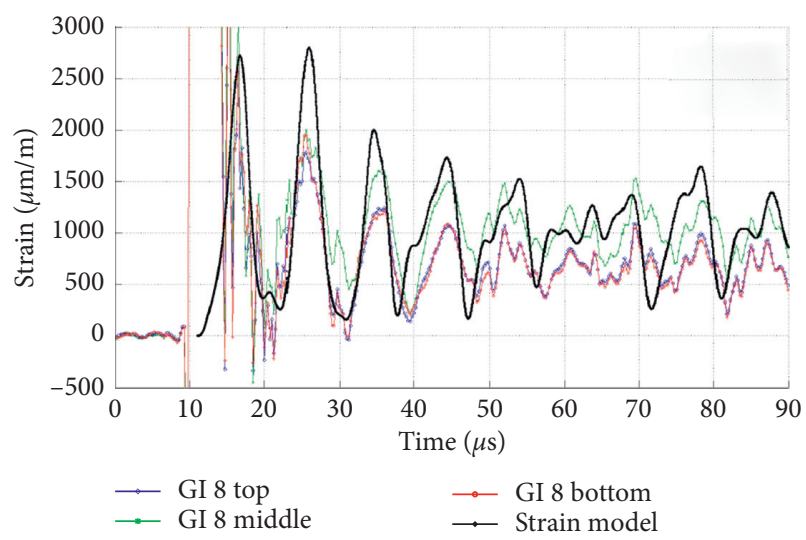

Figure 21: Results of the medium-intensity test on Glidcop ${ }^{\circledR}$, effect of the smoothing function on the hoop strain.

obtained during the test, and the simulated value. In this case, the impact of this difference is more relevant than in the medium-intensity measurements, because the spallation of the sample free face occurs at a distance of $2 \mathrm{~mm}$ from the beam axis and is therefore more dependent on the peaks of pressure and temperature achieved at the impact spot. One should, in particular, expect higher velocities of the spalls in the experimental case. However, the pulse length is also longer in the experimental case than in the numerical simulation, for the same total intensity ( 1.8 vs $1.5 \mu \mathrm{s})$. The power experimentally injected in the system is therefore lower than the simulated power, and this effect favors a higher spall velocity in the numerically simulated case.

Figure 26 shows the three Inermet ${ }^{\circledR} 180$ samples (numbered 1-2-3 from upstream to downstream in beam direction), observed from the high-speed camera dedicated window. A graduated grid covers the bottom of the tank and is also visible. The grid was used to calculate the speed front velocity by measuring the displacement of ejection between successive frames. As can be observed in Figure 26, a large quantity of the target material was ejected at high velocity from the two most loaded samples (no. 2 and 3); the high temperatures reached are confirmed by the intense light emitted by the fragments during a few hundred of microseconds. Numerical simulations predicted temperatures on the target exceeding $2000 \mathrm{~K}$, see Figure 27. At those temperatures, the $\mathrm{CuNi}$ matrix melts, while the tungsten particles are still in a solid state. It can also be observed that the light intensity produced by the molten material far exceeds the light, provided by the flash, which is already illuminating the system prior to the impact. 


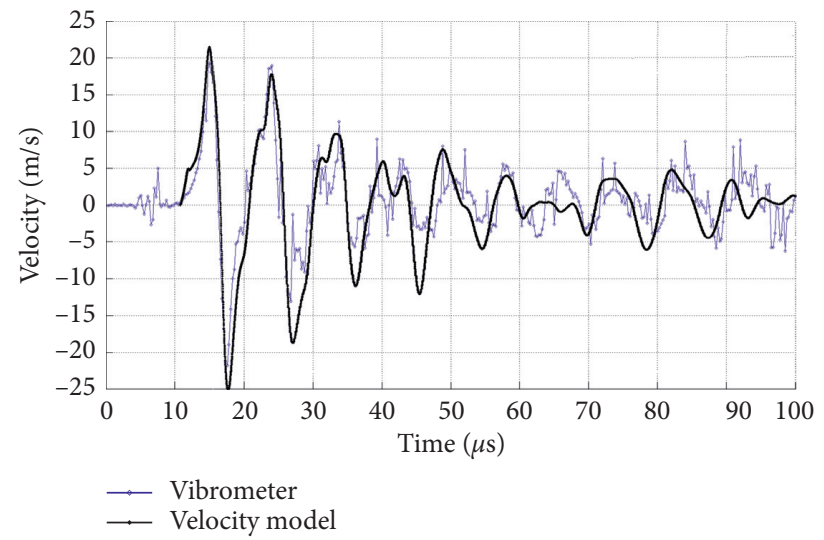

FIGURE 22: Results of the medium-intensity test on Glidcop ${ }^{\circledR}$, radial velocity without smoothing function. Acquired signal (blue) compared with simulated one (black).

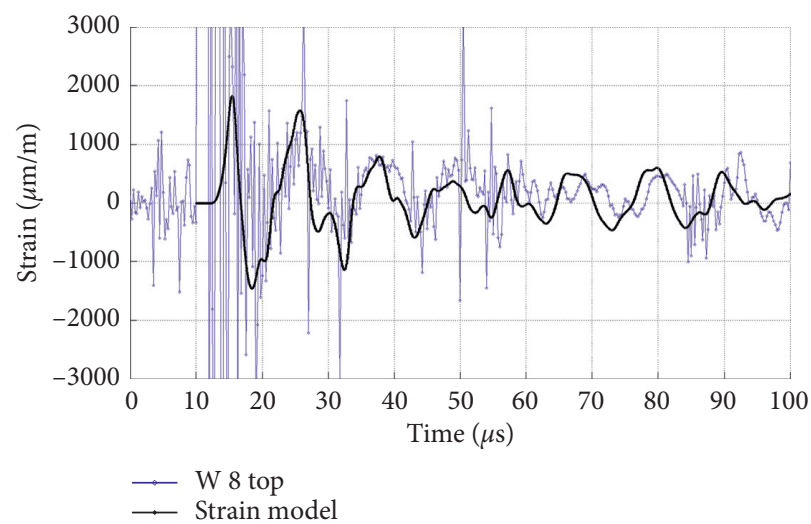

FIGURE 23: Results of the medium-intensity test on Inermet ${ }^{\circledR} 180$, acquired hoop strain (blue) without smoothing function and simulated signal (black).

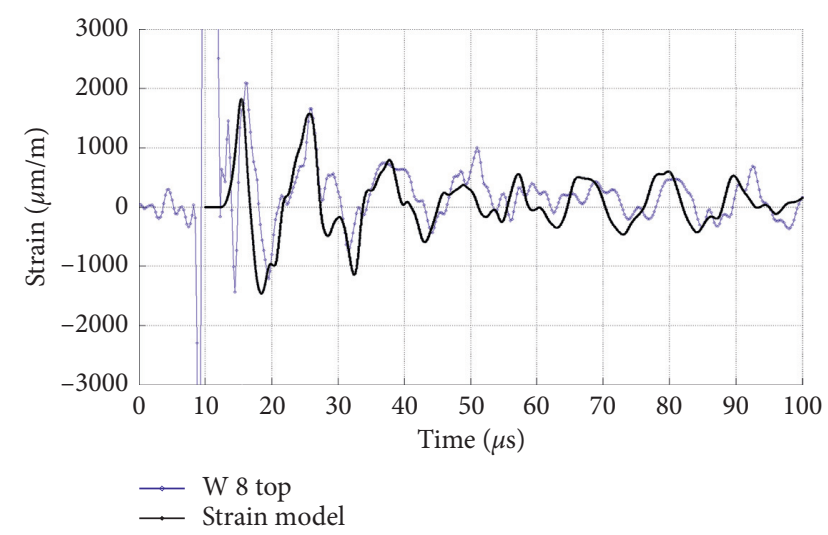

FIgURE 24: Results of the medium-intensity test on Inermet ${ }^{\circledR} 180$, acquired hoop strain (blue) with smoothing function and simulated signal (black).

Both ejected particle front shape and velocity are consistent with high-speed camera acquired data, Figure 28, even considering the differences between real and the simulated scenarios: the estimated velocity of the fragment front, as acquired, is $\sim 275(\mathrm{~m} / \mathrm{s})$, well matching the simulated velocity of $316 \mathrm{~m} / \mathrm{s}$, keeping in mind the

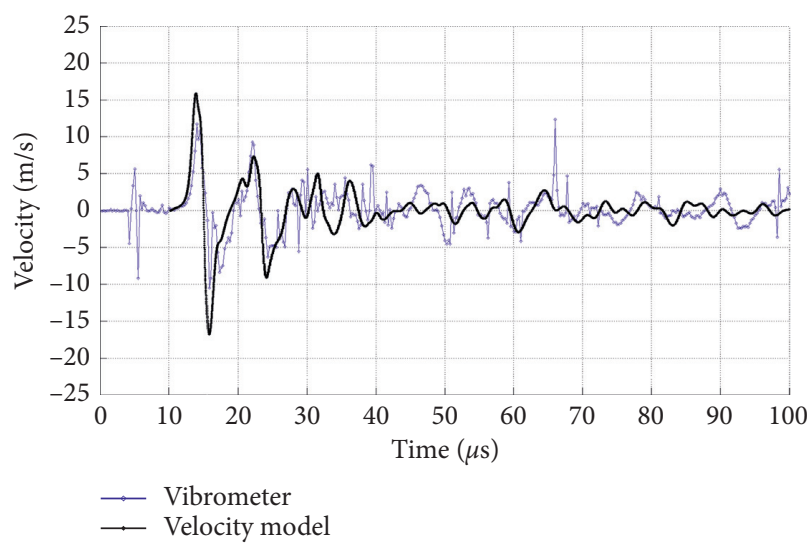

FIgURE 25: Results of the medium-intensity test on Inermet ${ }^{\circledR} 180$, radial velocity (blue) without smoothing function and simulated signal (black).

differences between numerical and experimental parameters discussed at the beginning of Section 6.3 (Figure 28).

The material ejected during the impact on the targets was deposited at the base of the tank and was clearly visible at the moment of the tank's opening, see Figure 29. 

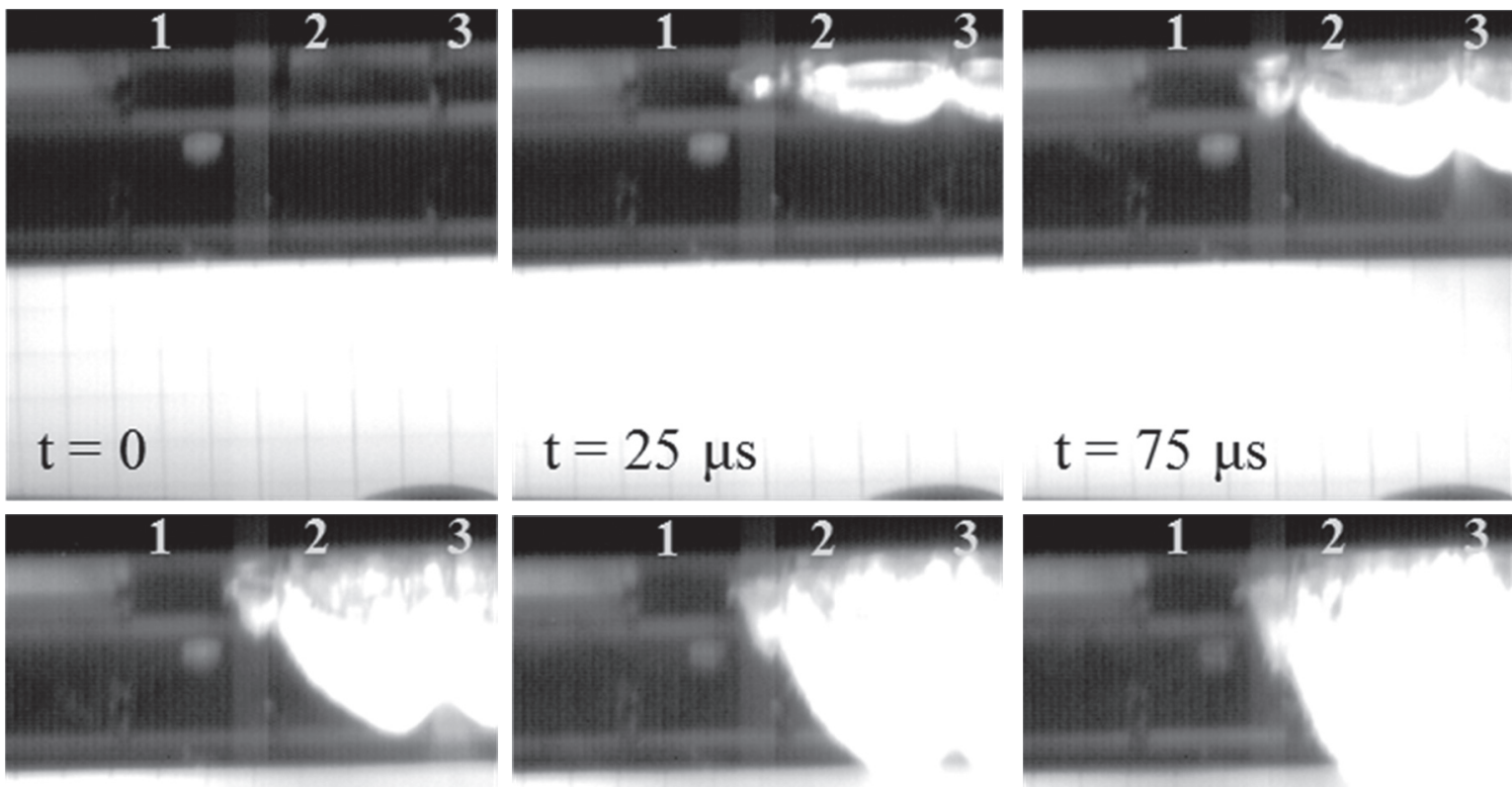

$$
\mathrm{t}=75 \mu \mathrm{s}
$$
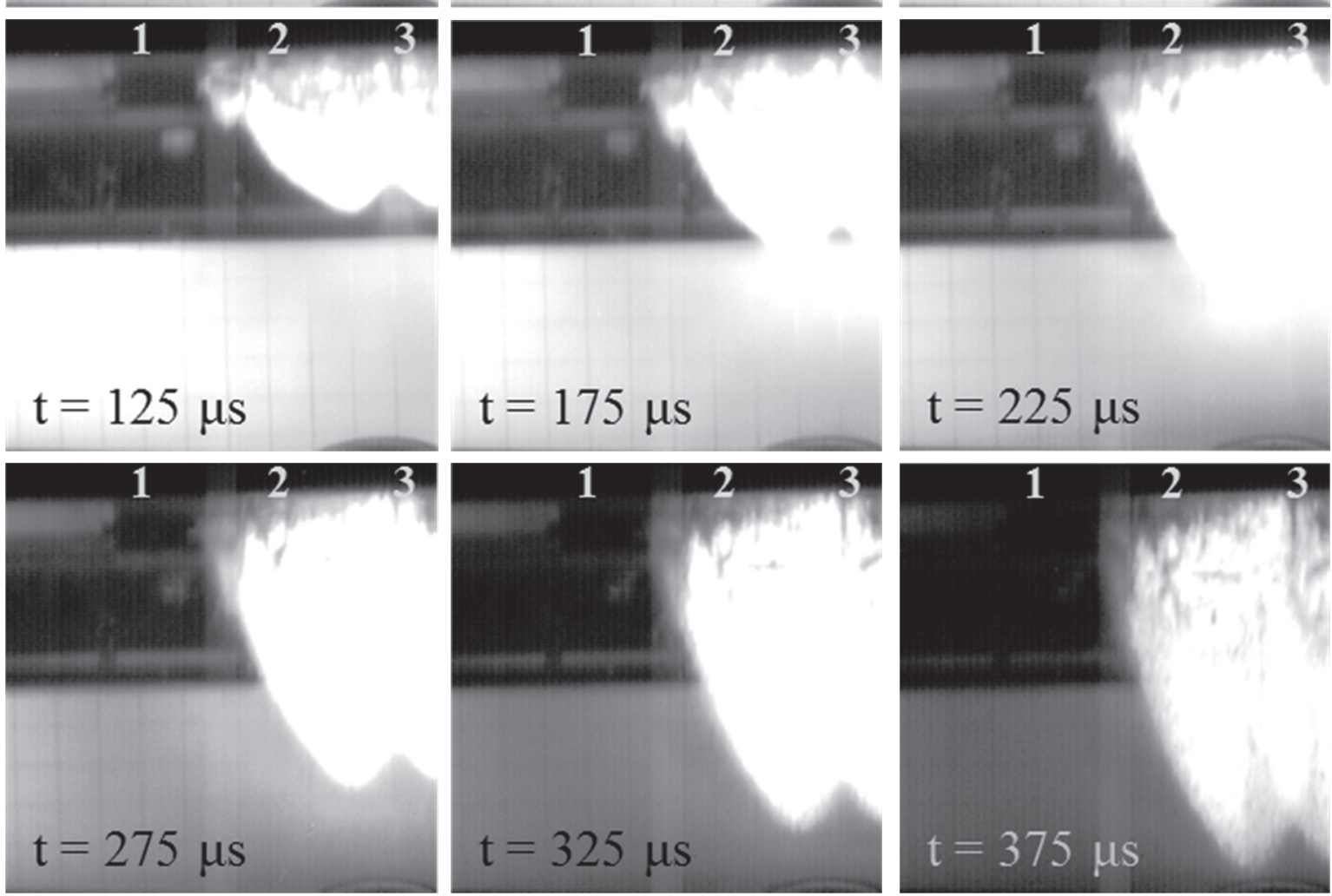

$$
\mathrm{t}=225 \mu \mathrm{s}
$$
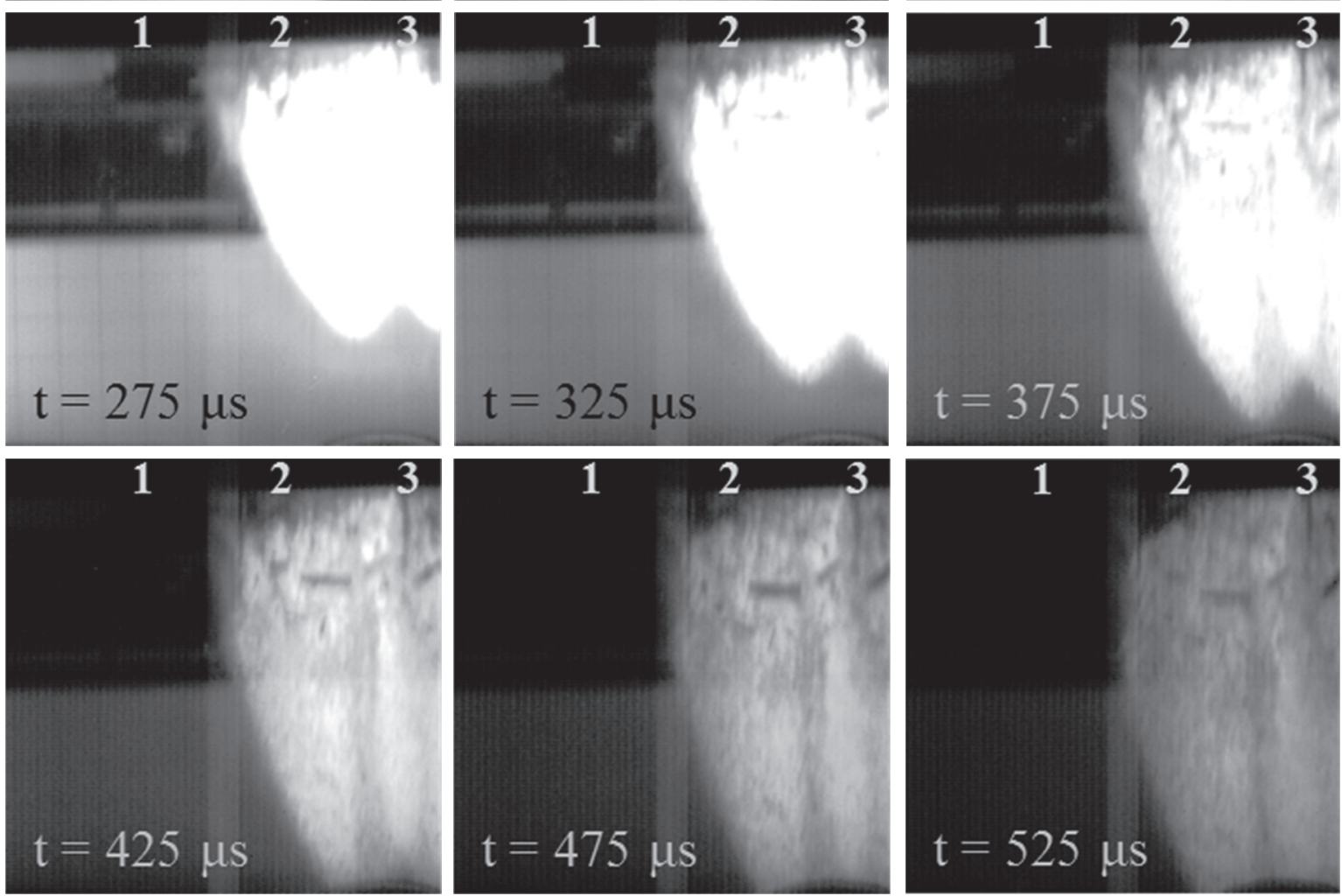

FiguRE 26: Image sequence of the impact on Inermet ${ }^{\circledR} 180$ of a 72 bunches proton pulse. Beam is coming from the left; three Inermet samples are partially visible (numbered 1 to 3) [29]. (a) $t=0$. (b) $t=25 \mu \mathrm{s}$. (c) $t=75 \mu \mathrm{s}$. (d) $t=125 \mu \mathrm{s}$. (e) $t=175 \mu \mathrm{s}$. (f) $t=225 \mu \mathrm{s}$. (g) $t=275 \mu \mathrm{s}$. (h) $t=325 \mu \mathrm{s}$. (i) $t=375 \mu \mathrm{s}$. (j) $t=425 \mu \mathrm{s}$. (k) $t=475 \mu \mathrm{s}$. (l) $t=525 \mu \mathrm{s}$. 


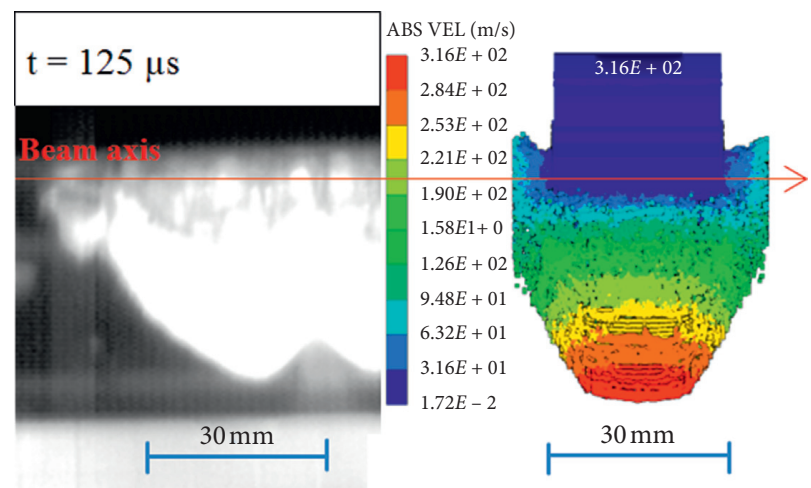

FIGURE 27: Temperature on the Inermet ${ }^{\circledR} 180$ target $25 \mu$ s after the high-energy impact. The ongoing material flow towards the surrounding is clearly visible, and the target deformations are in true scale.

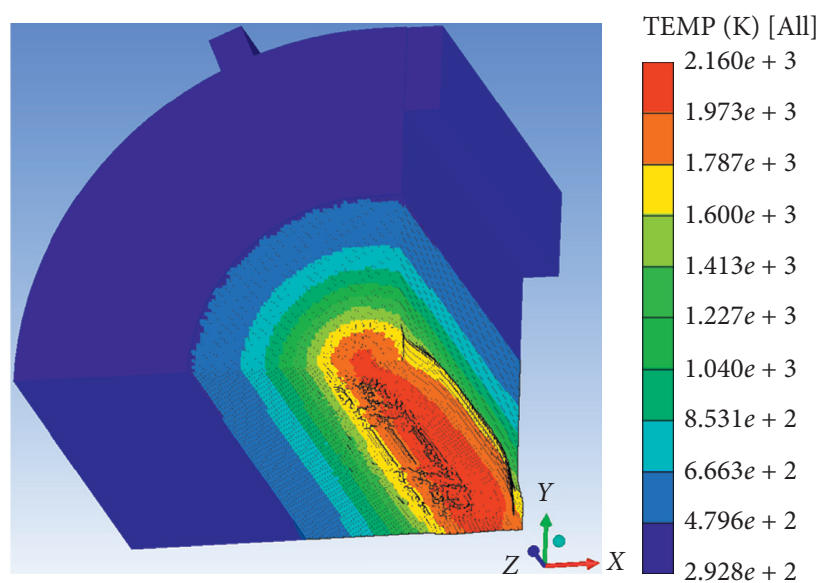

FIgURE 28: Comparison between simulation and acquired image $\sim 25 \mu \mathrm{s}$ after the impact.

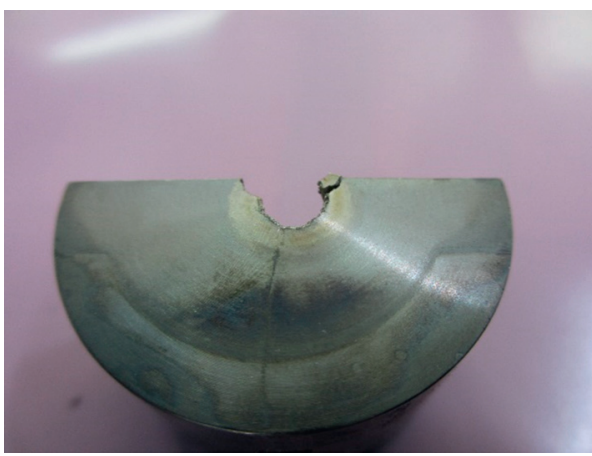

(a)

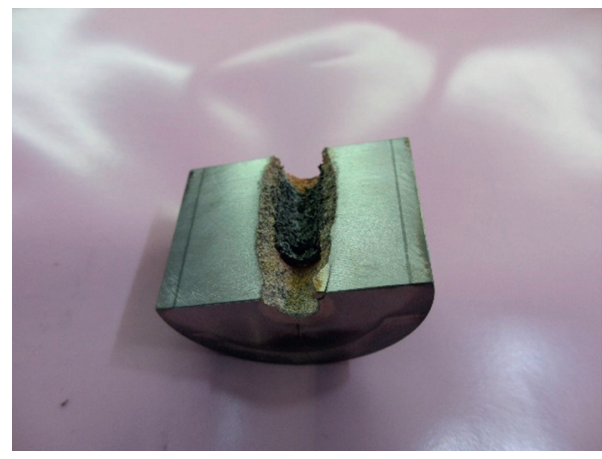

(c)

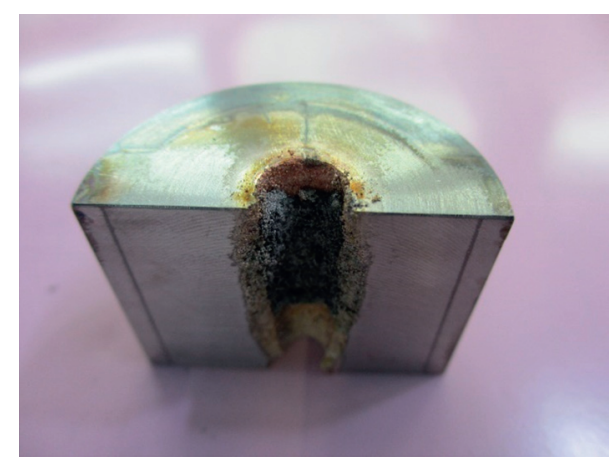

(b)

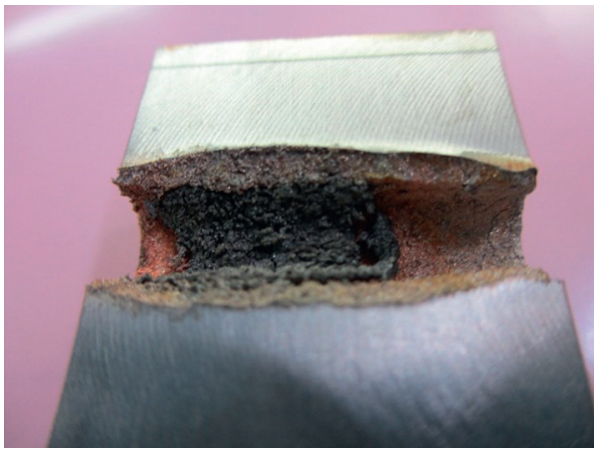

(d)

FIgURE 29: Views of the second Inermet ${ }^{\circledR} 180$ specimen after extraction from the HRMT-14 test bench. The crater created by the impact is elliptical, with vertical axis $9.4 \mathrm{~mm}$ and horizontal axis $8.7 \mathrm{~mm}$. The plastic deformation on the free surface also creates two "lips" spaced vertically by $13.3 \mathrm{~mm}[29]$. 


\section{Conclusions}

Studying the dynamic response of materials at high temperatures and strain rates calls for dedicated tests in facilities which employs accelerated particle or laser beams. In both cases, devising an ad hoc instrumentation is a challenging task, as the temperature and stresses produced by the beam at the impacted spot does not allow instrumenting that spot with elements such as temperature probes and strain gauges. The only viable solution is to use contact-less methods, such as LDVs and high-speed camera, and place the rest of the instrumentation at the closest possible distance from the impacted spot. On top of combining local and remote instrumentation to reconstruct the wave propagation phenomenon, the amplitude decay must be derived with special numerical simulations with codes such as Autodyn or LSDyna.

We presented a comprehensive method for allowing monitoring of the response of material targets to proton or ion beams at the CERN HiRadMat facility. This method was first used in the HRMT-14 experiments, and an overlook of the benchmarking of the acquired data with advanced simulations, performed with Autodyn, was given in this paper. The numerical aspects of the simulations, as well as more detailed sensitivity studies of the material models developed, are given in other dedicated papers such as $[14,27]$.

Before the construction of HiRadMat at CERN, previous tests were performed in target stations such as the TT40 tunnel, which makes use of the SPS proton beam. In such tests, however, also given to the space restrictions of the experimental area, the instrumentation was typically limited to a laser Doppler vibrometer [10]. The techniques developed and presented in this paper, on the other hand, allow a much wider monitoring of the thermomechanical response of targets hit by particle beams and may be of sure interest also for other tests where high strain rates and temperatures are involved, such as laser experiments, flyer-plate tests, and split-Hopkinson pressure test bars. All these tests involve temperatures which can easily attain peaks of $10.000 \mathrm{~K}$ and strain rates in the order of $106 \mathrm{~s}-1$ [29].

The HRMT-14 test also highlighted the strong electromagnetic coupling between the strain gauges and the particle beam, which typically lasts about $20 \mu \mathrm{s}$, hindering the first wave signal in the measurement. In successive HiRadMat experiments, such as for example HRMT-23 "Jaws" [12], optical fibres were added to the instrumentation system. In fact, optical fibres are immune to electromagnetic effects, as it has been also confirmed by recent works in harsh environments such as mercury target experiments [30]. This upgraded configuration will be the base of future experiments under proton beam impacts, such as the HRMT-57 "Multimat-2" test on composite material rods, planned at HiRadMat in late 2021.

\section{Data Availability}

The data used to support the findings of this study are available on CERN servers.

\section{Conflicts of Interest}

The authors declare that they have no conflicts of interest.

\section{Acknowledgments}

The research leading to these results has received funding from the European Commission under the FP7 Research Infrastructures project EuCARD-2, grant agreement no. 312453. This project has received funding from the European Union's Horizon 2020 Research and Innovation Program under grant agreement no. 730871. The authors wish to thank the HiRadMat facility team operators for the help and support provided during the research described in this paper.

\section{References}

[1] O. Bruning, P. Collier, P. Lebrun et al., The LHC Design Report, European Organization for Nuclear Research, London, UK, 2004.

[2] R. Schmidt, "Protection of the CERN large hadron collider," New Journal of Physics, vol. 8, pp. 1-31, 2006.

[3] A. Bertarelli, "The mechanical design for the LHC collimators," in Proceedings of the EPAC'04, pp. 545-547, Lucerne, Switzerland, 2004.

[4] R. Assmann, "Requirements for LHC collimation system," in Proceedings of the EPAC'02, pp. 197-199, Paris, France, 2002.

[5] S. Peggs, "The European spallation source," in Proceedings of PAC'11, pp. 2549-2551, New York, NY, USA, 2011.

[6] C. Accettura, Investigation of Radiation Damage Effect on $H L$ LHC Collimator Materials, Politecnico di Milan, Milan, Italy, 2013.

[7] M. Cauchi, "High energy beam impact tests on a LHC tertiary collimator at the CERN high-radiation to materials facility," Physical Review Accelerators and Beams, vol. 17, Article ID 021004, 2020.

[8] A. Bertarelli, "Updated robustness limits for collimator materials," Proceedings of MPP Workshop, vol. 108-112, 2013.

[9] R. Corrocher, F. Tedesco, P. Rabusin, and G. De Sandre, "Effect of human erythrocyte stromata on complement activation," British Journal of Haematology, vol. 29, 1975.

[10] A. Dallocchio, Study of Thermo-Mechanical Effects Induced in Solids by High Energy Particle Beams: Analytical and Numerical Methods, Politecnico di Torino, Turin, Italy, 2008.

[11] I. Efthymiopoulos, "HiRadMat: a new irradiation facility for material testing at CERN," in Proceedings of IPAC2011, pp. 1665-1667, San Sebastián, Spain, 2011.

[12] G. Gobbi, A. Bertarelli, F. Carra, J. Guardia-Valenzuela, and S. Redaelli, "Novel LHC collimator materials: high-energy Hadron beam impact tests and nondestructive postirradiation examination," Mechanics of Advanced Materials and Structures, vol. 27, no. 17, pp. 1518-1530, 2019.

[13] M. Pasquali, A. Bertarelli, C. Accettura et al., "Dynamic response of advanced materials impacted by particle beams: the MultiMat experiment," Journal of Dynamic Behavior of Materials, vol. 5, no. 3, pp. 266-295, 2019.

[14] M. Scapin, L. Peroni, A. Bertarelli, A. Dallocchio, N. Mariani, and S. Bizzaro, "Investigation of the behavior of metal-diamond composites," in Proceedings of ECCM15, Venice, Italy, 2012.

[15] A. Bertarelli, E. Berthome, V. Boccone et al., "An experiment to test advanced materials impacted by intense proton pulses 
at CERN HiRadMat facility," Nuclear Instruments and Methods in Physics Research Section B: Beam Interactions with Materials and Atoms, vol. 308, pp. 88-99, 2013.

[16] N. Charitonidis, "Radiological assessment of the collimator materials tests at hiradmat in 2012," 2020.

[17] F. Guarino, Compilation of Radiation Damage Test Data, European Organization for Nuclear Research, London, UK, 2011.

[18] A. Dräbenstedt, "Remote-sensing vibrometry at $1500 \mathrm{~nm}$ wavelength," 2012.

[19] G. A. Massey, "Study of vibration measurement by laser methods," 1968.

[20] B. Riemer, J. Haines, M. Wendel et al., "Cavitation damage experiments for mercury spallation targets at the LANSCEWNR in 2005," Journal of Nuclear Materials, vol. 377, no. 1, pp. 162-173, 2008.

[21] C. Rembe, Optical Inspection of Microsystems, Wolfgang Osten, London, UK, 2007.

[22] DIN EN 60825-1 VDE 0837-1:2015-07, Safety of Laser Products Part 1: Equipment Classification and Requirement.

[23] A. H. Johnson, "Radiation damage in electronic and optoelectronic devices in space," 2000.

[24] B. Hopkinson, "A method of measuring the pressure produced in the detonation of explosives or by the impact of bullets," Philosophical Transactions of the Royal Society of London, Ser. A, vol. 213, pp. 437-456, 1914.

[25] J. Simplicio, K. Schwenzer, and F. Maenpa, "Kinetics of cyanate and imidazole binding to hemin in micelles," Journal of the American Chemical Society, vol. 97, 1975.

[26] W. S. Cleveland and C. Loader, Smoothing by Local Regression: Principles and Methods, Springer, Berlin, Germany, 1996.

[27] A. Bertarelli, "First results of an experiment on advanced collimator materials at CERN HiRadMat Facility," in Proceedings of IPAC'13, Shanghai, China, 2013.

[28] A. Bertarelli, "Research and development of novel advanced materials for next-generation collimators," 2011.

[29] F. Carra, Thermomechanical Response of Advanced Materials Under Quasi Instantaneous Heating, Politecnico di Torino, Turin, Italy, 2017.

[30] Y. Liu, W. Blokland, C. Long, B. Riemer, M. Wendel, and D. Winderc, "Strain measurement in the spallation target using high-radiation-tolerant fiber sensors," IEEE SEnsors Journal, vol. 18, no. 9, pp. 3645-3653, 2018. 